\title{
Effect of low angle grain boundaries on morphology and variant selection of grain boundary allotriomorphs and Widmanstätten side-plates
}

\author{
D. Qiu ${ }^{\text {a, b }}$, R. Shi ${ }^{\text {b }}$, P. Zhao ${ }^{\text {b }}$ D. Zhang ${ }^{\text {a }}$, W. Lu ${ }^{\text {a, }}$, Y. Wang ${ }^{\text {b,* }}$ \\ ${ }^{a}$ State Key Laboratory of Metal Matrix Composites, Shanghai Jiao Tong University, 800 \\ Dongchuan Road, Shanghai 200240, People's Republic of China \\ ${ }^{\mathrm{b}}$ Department of Materials Science and Engineering, The Ohio State University, 2041 College \\ Road, Columbus, OH 43210, USA \\ *Corresponding authors: luweijie@ sjtu.edu.cn (W. Lu) and wang.363@osu.edu (Y. Wang)
}

\begin{abstract}
:
Morphology and variant selection (VS) of grain boundary (GB) allotriomorphs and Widmanstätten side-plates of $\alpha$ phase in an $\alpha / \beta$ titanium alloy, Ti-6Al-4V (wt.\%), are investigated using a three-dimensional phase field model. The structures of low-angle GBs (misorientation $\theta_{m} \leq 10^{\circ}$ ) are modeled as discrete dislocation networks using Frank-Bilby theory. It is shown that $\alpha$ allotriomorphs and side-plates compete with each other during precipitation and the final morphology and selected $\alpha$ variants exhibit a strong correlation with the GB dislocation structures. While the side-plate morphology is more preferred by a symmetrical tilt GB with $\theta_{m} \sim 10^{\circ}$, it can also be induced by a pure twist GB with $\theta_{m} \leq 5^{\circ}$. Quantitative analysis indicates that precipitate morphology and VS are determined by the interplay among (i) elastic interaction between a nucleating $\alpha$ precipitate and the GB dislocation networks, (ii) growth anisotropy determined by the relative inclination of the habit plane with respect to the GB dislocations, (iii) density of nucleation sites for the same variant and coalescence during growth, and (iv) spatial confinement from simultaneously nucleated neighboring $\alpha$ variants of dissimilar types.
\end{abstract}

Keywords: heterogeneous nucleation; dislocation; elastic interaction; transformation texture; phase field simulation 


\section{Introduction}

Extended defects such as grain boundaries (GBs) and dislocations are found to have profound influences on the nucleation and growth of precipitates in a complex manner that leads to drastically different microstructures and micro-textures and, consequently, considerable variations in mechanical properties [1-4]. In $\alpha / \beta$ titanium (Ti) alloys, for example, two distinctive types of $\alpha$ morphologies are commonly observed in $\beta$-processed alloys during cooling due to the presence of GBs, i.e., GB allotriomorphs (GB $\alpha$ ) and Widmanstätten side-plates (WS) [5-9]. Upon cooling from above the $\beta$ transus, GB $\alpha$ first nucleate and decorate the pre-existing $\beta$ GBs as either continuous layers $[10,11]$ or a group of small near-equiaxed particles of alternating variants [12]. On the other hand, it has been reported that WS may emanate from GB $\alpha$ that have already nucleated at $\beta$ GBs $[3,12,13]$. For example, after the GB $\alpha$ have occupied the GBs, WS of different orientations may nucleate and grow from the $\beta / \mathrm{GB} \alpha$ interfaces $[5,7,14]$. In other cases [15-17], the WS may even inherit the crystallographic orientation of the GB $\alpha$. However, it was also observed $[8,18]$ that discrete WS could grow directly from bare $\beta$ GBs without the presence of $\mathrm{GB} \alpha$. Therefore, apart from the effects of $\mathrm{GB} \alpha$, the intrinsic structural characteristics of $\beta$ GBs may also have a direct influence on the nucleation and growth of WS. In general, it is believed that the orientation and morphology of $\alpha$ precipitates at or near $\beta$ GBs depend strongly on the GB structures [19-21]. Furthermore, both GB $\alpha$ and WS could have pronounced influences on subsequent microstructural evolution and micro-texture development of $\alpha$ phase in the interiors of $\beta$ grains $[8,10,16,21]$.

It has been reported that the presence of continuous $\mathrm{GB} \alpha$ layers at GBs has a deleterious effects on properties, especially tensile ductility $[22,23]$. In contrast, discrete GB $\alpha$ particles of dissimilar orientation variants would lead to the subsequent formation of $\alpha$ colonies [23]. High density of boundaries between the Widmanstätten colonies of different orientations will shorten the effective slip length and result in tortuosity of crack path, leading to higher yield strength and high cycle fatigue strength $[13,22]$. Thus, the morphologies of the GB $\alpha$ and WS have direct impacts on the mechanical properties of Ti-alloys. It is therefore highly desirable to understand how GB structure affects the development of GB $\alpha$ and WS microstructure during precipitation [10]. 
According to the Burgers orientation relationship (OR) between $\beta$ and $\alpha$, i.e., $\{0001\}_{\alpha} \|\{110\}_{\beta}$ and $\langle 11 \overline{2} 0\rangle_{\alpha} \|\langle 111\rangle_{\beta}$ [24], twelve crystallographically equivalent orientation variants of the $\alpha$ phase could in principle precipitate out within one single $\beta$ grain [25]. From experimental observations, however, it often appears that only a subset of the twelve variants dominates the developed microstructure [26-29]. This phenomenon is known as variant selection (VS) and it has a significant effect on the mechanical properties (in particular, fatigue) of the alloy [30, 31]. Extensive studies have been carried out to investigate VS of GB $\alpha$ and WS due to pre-existing $\beta$ GBs $[8,9,11,21,32]$ and a set of empirical rules have been proposed based on experimental observations and some intuitive understanding of the possible mechanisms of the $\beta$ to $\alpha$ transformation. These rules include (i) GB $\alpha$ tend to select a variant that makes itself appear in an orientation that maintains the Burgers OR with both adjacent $\beta$ grains $[3,8,21,33$, 34], (ii) the angle between the low-energy interfaces of GB $\alpha$ (i.e., their habit planes) and the GB plane (GBP) should be as small as possible [27], (iii) the growth direction of GB $\alpha$ should be contained in the GBP $[33,34]$ and (iv) GB $\alpha$ will stay in an orientation that gives rise to the minimum interfacial energy with the non-Burgers $\beta$ grain through the "edge-to-edge" matching of the $\{110\}_{\beta}$ and $\{0002\}_{\alpha}$ planes [34]. However, these empirical rules are frequently violated according to experimental observations $[8,11]$, suggesting that the underlying mechanisms of VS due to GBs are still not fully understood [3].

In parallel to the experimental studies, extensive theoretical modeling has also been carried out to investigate the effect of GB on precipitation. For example, the nucleation of GB $\alpha$ has been analyzed using the classical theory of heterogeneous nucleation [35-40]. More recently, the influence of GBs on the morphology and VS of GB $\alpha$ has been studied [40] by analyzing experimental characterization results against the empirical rules mentioned earlier. These studies essentially treated GBs as continuиm dividing surfaces with an excess boundary energy. In reality, however, GBs have internal discrete atomistic structures that influence the properties of GBs in a profound manner [41-46] and, as a consequence, the nucleation and growth behavior of GB precipitates may vary significantly from GB to GB even though they may have similar boundary energy. 
In this study, we investigate the effects of different types of low angle GBs on precipitation of $\mathrm{GB} \alpha$ and WS using three-dimensional (3-D) phase field simulations. Low-angle (with misorientation angle $\theta_{m} \leq 10^{\circ}$ ) symmetrical tilt and pure twist GBs are considered for simplicity but without losing generality. It builds upon our previous work [47-49] on the effect of individual dislocations on precipitation. By incorporating discrete dislocation structures of these GBs, whose stress fields vary significantly with the GB characteristics, diverse GB $\alpha$ and WS microstructures have been observed in the simulations and the morphologies of these precipitates and selected $\alpha$ variants exhibit a strong correlation with the GB dislocation structures. In the following sections, we first introduce the theories and methods, including the calculation of GB dislocation structures and the resulting stress field of GB dislocation networks (Section 2.1), as well as a brief introduction of the multi-phase field model that is extended from our previous work in order to consider an elastically inhomogeneous solid medium (Section 2.2). Simulation results on morphology and VS of GB $\alpha$ and WS are presented in Section 3 and discussed in Section 4. Major findings of the study are summarized in Section 5.

\section{Methods}

\subsection{Dislocation networks at low-angle grain boundaries and their stress fields}

Small angle and special GBs as well as semi-coherent interfaces have been modeled as discrete dislocation networks using the Frank-Bilby theory [50, 51], O-lattice theory [52, 53] or microscopic phase field microelasticity theory [54]. The dislocations are called intrinsic dislocations in the sense that they inherently describe the structure of GBs and interfaces in an otherwise homogeneous continuum medium. In the current work, we apply the Frank-Bilby theory to describe GB dislocation networks. As a generic study focusing on the effect of discreteness in GB structures, symmetrical tilt and pure twist GBs consisting of (at most) two independent sets of dislocations are considered, with the line directions being $\xi_{1}$ and $\xi_{2}$ and the Burgers vectors being $\mathbf{b}_{1}$ and $\mathbf{b}_{2}$ [46, 55]. Following the Frank-Bilby equation [46], the net Burgers vector $\mathbf{B}$ across a GB between two adjacent grains having a misorientation angle, $\theta_{m}$, is given by:

$$
\mathbf{B}=2(\mathbf{p} \times \hat{\boldsymbol{\rho}}) \sin \left(\frac{\theta_{m}}{2}\right)=\left(\mathbf{N}_{1} \cdot \mathbf{p}\right) \mathbf{b}_{1}+\left(\mathbf{N}_{2} \cdot \mathbf{p}\right) \mathbf{b}_{2} .
$$

The first equation in (1) gives the definition of the net Burgers vector through a Burgers circuit 
across the GB, where $\mathbf{p}$, the so-called probe vector, is an arbitrary vector contained in the GBP and $\hat{\boldsymbol{\rho}}$, a unit vector along the rotation axis. The net Burgers vector is then decomposed into physically meaningful Burgers vectors of the reference crystal (i.e., the elementary dislocations in a b.c.c crystal) according to the second equation in (1). Here $\mathbf{N}_{i}=\frac{\mathbf{n} \times \boldsymbol{\xi}_{i}}{d_{i}}(i=1,2), \mathbf{n}$ is the GBP normal, $d_{i}$ is the inter-dislocation spacing of the $i^{\text {th }}$ array, and $\mathbf{N}_{i} \cdot \mathbf{p}$ gives the number of dislocations intersected by $\mathbf{p}$, which belong to the $i^{\text {th }}$ array. Finding the complete stress field of GB dislocation networks requires the determination of dislocation configurations $\left\{\left(\boldsymbol{\xi}_{i}, \mathbf{b}_{i}, d_{i}\right)\right\}_{i=1,2}$ by solving Eq. (1). Note that the solution for Eq. (1) is not unique until an appropriate "reference state" is selected and additional physical consideration and assumptions are applied.

The choice of this reference state cannot be arbitrary. A general procedure of finding the desired reference state has been established in Ref. [56]. In the reference state, the perfect registry of the lattice is maintained across the interface, i.e., in the reference state the interface is coherent. Volterra dislocations are then introduced to relax the reference state to the final crystallographically and mechanically consistent "natural state" $[46,56]$. For both pure tilt and twist GBs that are to be studied in this work, it has been shown that the "median lattice" is the desired reference state that satisfies the crystallographic constraints and is free of far-field stresses $[55,56]$. Once the reference state is determined, two sets of Volterra dislocations with Burgers vectors $\mathbf{b}_{1}$ and $\mathbf{b}_{2}$ will be introduced, and the corresponding $\xi_{i}$ and $d_{i}$ in Eq. (1) can be determined so long as the crystallographic degrees of freedom (DOFs) are specified [56]. Then by employing Fourier transform, the stress equilibrium equations can be solved using the spectral method and the resulting bi-periodic distortion tensor as well as the stress field can be obtained for elastically anisotropic media (see Ref. [56] for details).

\subsection{Phase-field model}

A three-dimensional multi-phase-field model for an elastically and structurally inhomogeneous system [57] is employed to simulate microstructural evolution and variant selection of $\alpha$ precipitates during $\beta \rightarrow \alpha+\beta$ phase transformation in bi-crystalline Ti-6Al-4V (wt.\%). In this approach, an arbitrary $\alpha+\beta$ microstructure is characterized by two concentration fields (i.e., 
conserved order parameters) $\left\{X_{i}(\mathbf{r})\right\}_{i=A l, V}$ and twelve structural order parameters (non-conserved), $\left\{\eta_{p}(\gamma, \mathbf{r})\right\}_{p=1}^{12} \quad\left(\gamma=\beta_{1}, \beta_{2}\right)$, within each grain. Both the conserved and non-conserved order parameters vary continuously across the $\alpha / \beta$ interfaces. The phase field model associated with the precipitation process has been used in our previous works $[25,57,58]$ and the methodology of incorporating the stress field due to GB dislocation networks is essentially the same as the case of incorporating the stress field of a single dislocation [49] except that the inhomogeneity in elastic constants are considered. Therefore, we omit here the model framework and formulation that can be found in the literature (e.g., chemical free energy [59], elastic energy of elastically inhomogeneous solid $[60,61]$ and kinetic governing equations of multi-phase-field $[62,63])$ and only specify the definition of the elastic interaction energy between a nucleating $\alpha$ precipitate of the $p^{\text {th }}$ variant in the $\gamma$ grain and a GB dislocation network:

$$
e_{\mathrm{GB}}^{\mathrm{int}}(\mathbf{r}, \gamma, p)=-\sigma_{i j}^{\mathrm{GB}}(\mathbf{r}) \varepsilon_{i j}^{00}(\gamma, p) \text {, }
$$

where $\sigma_{i j}^{\mathrm{GB}}(\mathbf{r})$ is the stress field associated with the GB dislocation network (see Section 2.1) and $\varepsilon_{i j}^{00}(\gamma, p)$ is the stress-free-transformation-strain (SFTS) tensor of the $p^{\text {th }}$ variant of a coherent $\alpha$ particle nucleating in the $\gamma$ grain. Equation (2) will be used to analyze the simulation results in Section 4.

\section{Results}

\subsection{Grain boundary dislocation networks}

As schematically shown in Fig. 1(a), starting with the reference state i.e., the median lattice that is essentially a perfect $\beta$ crystal, the symmetrically tilted bi-crystal is obtained by first halving the (infinite) perfect crystal along the $(010)_{\beta}$ plane and rotating the two resulting grains by $\theta_{m} / 2$ and $-\theta_{m} / 2$, respectively, around an axis parallel to the $[101]_{\beta}$ direction. Since slip in b.c.c metals usually occurs in the close-packed $\langle 111\rangle_{\beta}$ directions with the Burgers vector of dislocations being $a_{\beta} / 2\langle 111\rangle_{\beta}$ ( $a_{\beta}$ is the lattice parameter of the b.c.c $\beta$ phase) [64], we select the only two independent Burgers vectors $\mathbf{b}_{1}=a_{\beta} / 2[\overline{1} 11]_{\beta}$ and $\mathbf{b}_{2}=a_{\beta} / 2[11 \overline{1}]_{\beta}$ within the $(101)_{\beta}$ plane as the inputs to obtain the GB dislocation structure. Once the Burgers vectors are determined, the nature of the dislocations (the sense vector $\xi_{1}$ and $\xi_{2}$ ) and the geometry of the 
dislocation configuration (the spacing $d_{1}$ and $d_{2}$ ) are solved using Eq. (1), which are listed in Fig. 1. According to the calculation based on the Frank-Bilby equation (Eq. (1)), the sense vectors and inter-dislocation spacing of the two sets of dislocations for a symmetrical tilt GB are identical, i.e., $\xi_{1}=\xi_{2}=[101]_{\beta}$ and $d_{1}=d_{2}=\frac{\mathbf{b}_{1}+\mathbf{b}_{2}}{2 \sin \left(\theta_{m} / 2\right)}=\frac{\mathbf{b}}{2 \sin \left(\theta_{m} / 2\right)}\left(\mathbf{b}=\mathbf{b}_{1}+\mathbf{b}_{2}=a_{\beta}[010]_{\beta}\right)$. Thus the two dislocation arrays characterized by $\left\{\xi_{1}, \mathbf{b}_{1}, d_{1}\right\}$ and $\left\{\xi_{1}, \mathbf{b}_{1}, d_{1}\right\}$ can actually be represented equivalently (in terms of the stress field) by a single array characterized by $\{\xi, \mathbf{b}, d\}$ with $\mathbf{b}=\mathbf{b}_{1}+\mathbf{b}_{2}=a_{\beta}[010]_{\beta}, \xi=\xi_{1}=\xi_{2}$ and $d=d_{1}=d_{2}$ as shown in Fig. 1(a). In other words, the stress field associated with the dislocation array of $\{\boldsymbol{\xi}, \mathbf{b}, d\}$ is identical to the superposition of that of $\left\{\xi_{1}, \mathbf{b}_{1}, d_{1}\right\}$ and that of $\left\{\xi_{2}, \mathbf{b}_{2}, d_{2}\right\}$. The pure twist boundary is obtained following the same procedure except for halving the crystal along the $(101)_{\beta}$ plane, which leads to two sets of equally spaced dislocations intersecting with each other, with Burgers vector $\mathbf{b}_{1}=a_{\beta}$ / $2[\overline{1} 11]_{\beta}$ and $\mathbf{b}_{2}=a_{\beta} / 2[11 \overline{1}]_{\beta}$, respectively, as shown in Fig. 1(b).

Our current treatment of approximating the grain boundary structure with two dislocation arrays satisfying the Frank-Bilby equation has been justified recently by Vattré et al. [65] using direct atomistic results for twist boundary with misorientation angle of $0^{\circ} \sim 10^{\circ}$. According to Ref. [65], the results from the continuum approach (used in our current work) show that the interfacial energy and hence the underlying stress field can be drastically different for different dislocation configurations selected. Since the way of calculating the interfacial energy in the atomistic approach is quite different from that in the continuum approach, an agreement of the two approaches on the interfacial energy calculation [65] suggests that the selected dislocation configuration (satisfying the Frank-Bilby equation) can well represent the atomistically stable interface (grain boundary) structure and the contribution due to dislocation reaction can be safely ignored as a first-order approximation. In Ref. [65], there are three independent elementary dislocations available to choose and thus all possible combinations need to be considered to determine the one that can well represent the atomistically calculated interface structure. In our current case, only two independent elementary dislocations (as discussed above), which are widely observed in experiments, are present, giving a unique dislocation configuration that satisfies the Frank-Bilby equation. 
The two sets of dislocations comprising the twist boundary are mainly of screw characters but also with certain edge components. Such calculated non-orthogonal dislocation configuration is exactly the same as that proposed by Hirth et al. [55]. While it is possible that the deviation from being orthogonal can lead to local dislocation nodal reaction, we believe that the consequence can only bring certain local core structure variations at the atomistic scale and the dislocation configuration predicted by the Frank-Bilby equation should still represent reasonably well an atomistically stable grain boundary, according to the results of Vattré et al. [65]. It is such an approximation that allows us to study the long-range influence of grain boundaries using the continuum elasticity theory, which is similar to assigning a cut-off radius for dislocation cores in the continuum elasticity theory of dislocations, and is usually considered legitimate when the focus is on the long-range stress field related phenomena. Being aware of this assumption, we will discuss further about its potential influence on the dislocation configurations and possible improvement in Section 4.5.

\subsection{Morphology and variant selection of $\alpha$ precipitates at symmetrical tilt $\beta$ grain boundaries}

The stress fields associated with symmetrical tilt GBs with different $\theta_{m}$ are first calculated according to the method described in Section 2.1 and then incorporated into the phase field simulations to study their influences on $\alpha$ precipitation at or near GBs. The development of different variants of $\alpha$ precipitates on both sides of the GBs with $\theta_{m}=3.5^{\circ} \sim 10^{\circ}$ at $\mathrm{T}=1070 \mathrm{~K}$ is shown in Fig. 2, where $\tau$ is a dimensionless time ( $\tau=1$ in the simulation corresponds to a real time of $\mathrm{t}=10^{-4} \mathrm{~s}$ ). Different variants of $\alpha$ precipitates are represented by different colors in the figures (Note that the color scheme is the same for variants in two adjacent $\beta$ grains). The GBP is represented by light gray plane with a thick white line at its edge. Langevin noises are turned on in the following phase-field simulations from $t=0$ to $t=1.67 \times 10^{-4} \mathrm{~s}$ with a dimensionless amplitude of 1.2 [66], which have equal influences on the nucleation of all $\alpha$ variants. The amplitude of noises is confirmed to a relatively low value such that the noise term only accounts for the inevitable chemical and structural fluctuations and the simulated morphology (in particular, at the nucleation stage) is still mainly driven by the stress field associated with GBs. (This is 
similar to our previous treatment in the studies of individual dislocations effect [49].)

For all cases studied, the nucleation of $\alpha$ precipitates takes place along the lines of dislocations, as shown in Figs. 2(a), (c), (e) and (g) at $\tau=0.5$. Note that the microstructures are represented by iso-surface contour plots with $\eta_{p}(\gamma, \mathbf{r}) \geq 0.5$. Before $\tau \leq 1$ when the noises are still on, since the amplitude of structural fluctuations produced by the noise term away from the GBP is much less than 0.5 , they are absent in these figures. Different $\alpha$ variants are induced by each individual dislocation line on both sides of the GBP and grow anisotropically. Over time, extensive coalescence (among the same variants) and impingement (among different variants) take place, mainly along the dislocation lines at the beginning and then in between the dislocation lines at later stages (in particular for large misorientations). Among the $\alpha$ precipitates, some of them (mainly those with their habit planes containing the dislocation lines) develop into laths with well-defined habit planes, emanating from the GBP and grow into the interior of the $\beta$ grains as typical WS structures (i.e., V7, V10 and V12 in Fig. 2(g)). The others remain as equiaxed GB $\alpha$ (i.e., V4 and V6 in Figs. 2(a), (c) and (e)) because their habit planes do not contain the dislocation lines and thus they cannot grow and coalesce along dislocation lines. Actually their growth is confined by their neighboring precipitates. Both WS and GB $\alpha$ are distributed periodically along the GBs due to the discreteness of GB dislocations.

As the misorientation increases, there is a gradual morphological transition of the $\alpha$ precipitates from a mixture of equiaxed GB $\alpha$ and isolated WS to mainly colonies of WS (Figs. 2(b), (d), (f) and (h)). As will be discussed in Section 4.3, such a morphological change originates from the underlying GB structure change. The $\alpha$ variants in the WS colonies at $\theta_{m}=10^{\circ}$, i.e., V7/V10/V12 in $\beta_{1}$ and V1/V9/V11 in $\beta_{2}$, have their habit planes parallel or nearly parallel to the dislocation lines and have a small inclination angle (only $\sim 8^{\circ}$ ) with each other. In each grain, any two of these three variants form an $\alpha / \alpha$ misorientation of the "D" type, i.e., $63.26^{\circ}$ rotation around $\left[\begin{array}{llll}10 & 5 & 5 & 3\end{array}\right]_{\alpha}$ [32]. Quantitative analysis and discussion on the crystallographic relationship between the selected $\alpha$ variants and the dislocation network will be presented in Section 4.2. 


\subsection{Morphology and variant selection of $\alpha$ precipitates at pure twist $\beta$ grain boundaries}

Different from the symmetrical tilt GB considered above, the twist GB consists of two sets of dislocation arrays intersecting with each other with an inclination angle of $\phi=70.5^{\circ}$, as shown schematically in Fig. 1(b). Precipitation at the twist GBs with $\theta_{m}=2^{\circ}, 3^{\circ}, 5^{\circ}$, and $10^{\circ}$ are investigated. Note that when $\theta_{m}$ changes, only the inter-dislocation spacing changes and $\phi$ remains constant. Figure 3 shows the morphology of $\alpha$ precipitates at GBs (as indicated by the light gray planes and white lines) with different $\theta_{m}$ from three different viewing directions at $\tau=10$. It is readily seen that in the case of $\theta_{m}=2^{\circ}$, variants in $\beta_{1}$ and $\beta_{2}$ grow along the dislocation line $\xi_{1}$ and $\xi_{2}$, respectively, i.e., the precipitates follow just one set of the parallel dislocation lines on each side of the GBP. Among all the precipitates, variants V3/V5/V9/V11 dominate in $\beta_{1}$ and V4/V6/V10/V12 dominate in $\beta_{2}$. In particular, while V3/V5 $\left(\beta_{1}\right)$ and V4/V6 $\left(\beta_{2}\right)$ grow nearly parallel to (i.e., spread over) the GBP on each side and form GB $\alpha$ layers, V9/V11 $\left(\beta_{1}\right)$ and V10/V12 $\left(\beta_{2}\right)$ grow nearly perpendicular to the GBP and form WS. This is because their habit planes are nearly parallel or perpendicular to the GBP, respectively.

When the misorientation angle increases, more WS are formed along the dislocations in both $\beta$ grains (Figs. 3(b)-(c)). At the same time, two different variants, i.e., V2 and V8, in both $\beta_{1}$ and $\beta_{2}$ start to spread between the WS and form GB $\alpha$ layers at $\theta_{m}=5^{\circ}$, replacing the GB $\alpha$ layers of V3/V5 in $\beta_{1}$ and V4/V6 in $\beta_{2}$ observed in the case of $\theta_{m}=2^{\circ}$ and $\theta_{m}=3^{\circ}$. For the sake of convenience, we denote GB $\alpha$ layer at lower misorientation angle consisting of V3/V5 $\left(\beta_{1}\right)$ or V4/V6 $\left(\beta_{2}\right)$ as Layer-I and that at higher angle consisting of V2/V8 as Layer-II. In the case of the largest misorientation $\theta_{m}=10^{\circ}$ considered, the majority of the GB on both sides is covered by $\mathrm{GB} \alpha$ Layer-II.

The time evolution of $\alpha$ precipitates in $\beta_{1}$ with $\theta_{m}=2^{\circ}$ and that in $\beta_{2}$ with $\theta_{m}=10^{\circ}$ are shown in Figs. 4(a)-(d) and (e)-(h), respectively. In the case of $\theta_{m}=2^{\circ}, \alpha$ precipitates first nucleate as discrete particles along the dislocation lines at $\tau=1$, as shown in Fig. 4(a). During the subsequent evolution, the particles grow and impinge upon or coalesce with each other to form extended GB $\alpha$ Layer-I (e.g., V3 by the circles) or WS (e.g., V9 by black arrows). In the case of 
$\theta_{m}=10^{\circ}$, there is obviously a more uniform distribution of $\alpha$ precipitates on the GBP because of the smaller spacing between dislocations. Similar growth, impingement and coalescence processes lead to GB $\alpha$ Layer-II and WS, but with one clear distinction, i.e., the coalescence of $\alpha$ variants into $\mathrm{GB} \alpha$ layer exhibits not only 1-D extension along the line directions of both dislocation arrays (highlighted by the white ellipses in Figs. 4(f)-(h)), but also 2-D extension across different dislocation lines, as indicated by the yellow circles.

Comparison between precipitation at symmetrical tilt (Fig. 2) and twist (Figs. 3 and 4) boundaries suggests that the morphology of GB $\alpha$ and WS and the corresponding tendency to form such morphologies vary significantly with the type of GBs and the misorientation angle. For instance, the $\mathrm{GB} \alpha$ could appear either as discrete equiaxed $\mathrm{GB} \alpha$ (at $3.5^{\circ}$ tilt $\mathrm{GBs}$ ) or as extended $\mathrm{GB} \alpha$ layer (at $10^{\circ}$ twist GB) and the WS could be either isolated side-plates (near $5^{\circ}$ twist $\mathrm{GBs}$ ) or side-plate colonies (near $10^{\circ}$ tilt GBs). Furthermore, even the type of variants constituting the GB $\alpha$ layers can vary with $\theta_{m}$, as has been shown in the cases of twist GBs.

\subsection{Two-dimensional cross sections}

Because most of the experimental observations of GB $\alpha$ are made on two-dimensional (2-D) cross sections, some special 2-D cross sections are obtained from the 3-D images of twist GBs presented above. In Figs. 5(a) and (b), the cross sections are chosen such that one of the two dislocation arrays are parallel to the cross section planes for the case of $\theta_{m}=2^{\circ}$ at $\tau=5$. In these cross sections, some of the $\mathrm{GB} \alpha$ precipitates appear as a continuous layer consisting of a single variant (see, e.g., the one indicated by the big white arrow in Fig. 5(d)), which looks like being at the onset of Mullins-Sekerka interface instability $[67,68]$ that leads to the development of WS colony at later stages $[69,70]$. Some others appear as discrete particles consisting of alternating variants, as indicated by the big white arrows in Fig. 5(e) (note that if different variants are not distinguished, these $\mathrm{GB} \alpha$ precipitates may also look like a continuous layer with developing interface instabilities); and some others appear as particles nucleated off the $\mathrm{GB} \alpha$, as indicated by the small red arrows. In Fig. 5(c) a similar cross section (Fig. 5(f)) is made for the case of $\theta_{m}=5^{\circ}$ at $\tau=10$, which shows that most of the $\alpha$ precipitates near the GB appear as WS grown directly off the GBP. All these GB $\alpha$ and WS morphologies have been observed frequently in 
experiments $[4,10,12,15,21,22,71,72]$. Note that only two types of special GBs are considered in the current study. If more GB types are considered, a lot more GB $\alpha$ morphologies on 2-D cross sections are expected.

\section{Discussion}

Till now, the mechanisms of VS by GBs remain controversial, including whether the selection process is nucleation- or growth-dominated [3]. In the current study, VS at the early nucleation stage is found to be driven mainly by the elastic interaction between the nucleating coherent $\alpha$ variants and the anisotropic and heterogeneous stress field associated with discrete GB dislocations. However, because of their mutual interactions and their interactions with the GB dislocations during growth, some of the variants grow much faster than the rest and some actually dissolve during subsequent evolution, which alter the final VS results. The change of misorienation angle will alter these interactions and hence affect the final VS results accordingly. In the following sections, the occurrence of VS and its evolution will be discussed separately for the nucleation and growth stages in cases with different misorientation angles, and the existing empirical rules of VS by GBs will be assessed against the simulation results.

\subsection{Effect of elastic interaction between a precipitates and GB dislocations during nucleation}

To analyze the effect of elastic interactions between nucleating $\alpha$ particles and GB dislocation networks, their interaction energy $e_{\mathrm{GB}}^{\mathrm{int}}(\mathbf{r}, \gamma, p)$ is calculated for different variants according to Eq. (2). The calculation shows that the interaction energy for each variant decays rapidly when moving away from the GBP. For example, the interaction energy of V1 at a distance of $1 / 2|\mathbf{b}|$ away from the GBP of the $3.5^{\circ}$ symmetrical tilt boundary on the side of $\beta_{2}$ is $-3262 \mathrm{~J} / \mathrm{mol}$ and drops to $-784 \mathrm{~J} / \mathrm{mol}$ at $2|\mathbf{b}|$. This indicates that the position of the maximum interaction energy for each variant will locate at the core regions (radius $=1 / 2|\mathbf{b}|$ ) of the dislocations. Of course this result is obtained using the Volterra dislocation model (sharp core) [73] employed in the current study. If the Peierls dislocation model (diffuse core) [74] is used, the location and magnitude of the maximum interaction may change. In Fig. 6 we plot the most negative interaction energy at the

core region, $e_{\min }^{\mathrm{int}}(\gamma, p)$, between each variant and the GB dislocations. Note that such interaction energy does not change greatly with the misorientation angle within the small range considered in 
the current study. Figure 6 shows that the same variant in different $\beta$ grains (i.e., $\beta_{1}$ and $\beta_{2}$ ) has similar (all negative) interaction energy, among which V3-V6 and V9-V12 in both grains have the most negative values (around $-6000 \mathrm{~J} / \mathrm{mol}$ ), indicating that $\alpha$ precipitates of these variants are the most favored ones during nucleation. However, by comparing the precipitate microstructures obtained for the tilt GBs with $\theta_{m}=3.5 \sim 10^{\circ}$ at the early stage ( $\tau=1$ ) (Fig. 2), we find that the type of variants and morphology of $\alpha$ precipitates at any individual dislocation do not vary with $\theta_{m}$ and the variants selected are not limited to those with the most negative interaction energy, $e_{\mathrm{min}}^{\mathrm{int}}$. For example, V7 in $\beta_{1}$ distributed along the dislocation lines in Fig. 2(e) actually has much less negative $e_{\mathrm{min}}^{\mathrm{int}}$ values. This leads to the following analysis of the position of $e_{\mathrm{min}}^{\mathrm{int}}$ for each variant and the corresponding influence on the precipitation process.

In Table 1 we list the position of $e_{\min }^{\mathrm{int}}$ around an edge dislocation of a symmetrical tilt GB for all the $\alpha$ variants in both $\beta_{1}$ and $\beta_{2}$, which is characterized by the inclination angle, $\omega$ (see the inset of Table 1), between the vectors $[010]_{\beta}$ and $\mathbf{r}$ with $e_{\min }^{\mathrm{int}}$. It shows that V2-V6 and V8-V12 have their preferred nucleation sites located at either exactly the same or nearby sites (e.g., at $\omega=236^{\circ}$ or $213^{\circ}$ in $\beta_{1}$ and $297^{\circ}$ and $315^{\circ}$ in $\beta_{2}$, which correspond to, respectively, the bottom left and right of the edge dislocation shown in the inset of Table 1). In contrast, V1 only shares the same preferred nucleation site with V7 (i.e., $\omega=135^{\circ}$ in $\beta_{1}$ and $37^{\circ}$ in $\beta_{2}$, i.e., the top left and top right of the edge dislocation). This has a significant consequence on the VS process during precipitation. For example, even though V7 and V1 do not have the most negative interaction energy (see Fig. 6), they have much fewer "competitors" for the same preferred nucleation sites as compared to those variants that have the most negative interaction energy (e.g., V3-V6 and V9-V12). As a result, V1 and V7 end up being the most dominant variants (with the largest volume fractions) in all cases of the tilt GBs from $\theta_{m}=3.5^{\circ}$ to $\theta_{m}=10^{\circ}$, as shown in Fig. 2. Thus if a unique location (i.e., a unique angle $\omega$ ) around the dislocation core is preferred by just a single variant, then many particles of this variant will nucleate at the same radial location along the dislocation line. They will coalesce with each other during growth. As will be discussed in the following section, if the habit plane of the variant is parallel to the GBP, it will have the maximum growth rate in the GBP and evolve into GB $\alpha$ layer. If its habit plane contains the dislocation line at which they nucleate, but has a relatively large angle of inclination with the GBP, 
it will grow into the interior of the $\beta$ grains without much interference and evolve into WS. In contrast, if many variants share the same preferred location around the dislocation core for nucleation, then many different variants will nucleate (due to the underlying stochastic nature) at the same radial location along the dislocation line. They impinge upon each other during growth and form more or less equiaxed $\mathrm{GB} \alpha$ due to the spatial confinements from neighboring variants.

Similar interaction energy calculations made for the twist GB of $\theta_{m}=2^{\circ}$ are plotted in Fig. 7(a). Opposite from that of the tilt GB case, the results show that V2 and V8 have much larger magnitudes of the interaction energy $e_{\min }^{\mathrm{int}}(\sim-6900 \mathrm{~J} / \mathrm{mol})$ than that of the remaining in both grains, followed by V3/V5/V9/V11 $\left(\beta_{1}\right)$ and V4/V6/V10/V12 $\left(\beta_{2}\right)$. The interaction energy fields, $e_{\mathrm{GB}}^{\text {int }}(\mathbf{r}, \gamma, p)$, of some selected variants in both grains are shown in Figs. 7(b)-(c) on a cross-section (parallel to GBP) at a distance of $2 / 3\left|\mathbf{b}_{1}\right|$ away from the GBP, where the preferred nucleation sites are also identified.

It is interesting to note that all the variants in $\beta_{1}$ exhibit negative interaction energy near dislocation $\xi_{1}$ and all variants in $\beta_{2}$ exhibit negative interaction energy near dislocation $\xi_{2}$. This is not surprising once we notice that both dislocation $\xi_{1}$ and $\xi_{2}$ are mainly screw type with the angle between the Burgers vector and sense vector (see Fig. 1(b)) being less than $20^{\circ}$. Since the stress field of a straight screw dislocation is of simple shear type and changes sign when moving from one side of the shear plane to the other [64], the most preferred nucleation sites switch to the other set of dislocations as shown in Figs. 7(b)-(c).

A closer examination shows that the preferred nucleation sites for V2 in both grains are limited to the intersections (nodal points, indicated by the rectangles) of the GB dislocation network, around which the interaction energy $e_{\mathrm{GB}}^{\mathrm{int}}$ reaches the most negative values, as indicated in the insets of Figs. 7(b)-(c), where the variations of $e_{\mathrm{GB}}^{\mathrm{int}}$ along the yellow arrows are shown. Because of the symmetry, V8 also has the same distribution of $e_{\mathrm{GB}}^{\mathrm{int}}$ (not plotted in Fig. 7) as that of V2. This explains the morphology shown in Fig. 4(e) where more nuclei of V2 and V8 form at $\theta_{m}=10^{\circ}$ compared with those at $\theta_{m}=2^{\circ}$ (Fig. 2(a)), due to the increase in dislocation density and hence 
in the density of nucleation sites (nodal points). Also, the particles tend to form well-aligned arrays set by the nodal points (Figs. 4(e) and (f)), which later spread over the GBP in both 1-D and 2-D modes (as indicated by the white ellipses and yellow circles, respectively). On the contrary, the locations of preferred nucleation sites (i.e., locations of $e_{\mathrm{min}}^{\mathrm{int}}$ ) associated with all the remaining variants such as V3/V5/V9/V11 in $\beta_{1}$ are distributed along the dislocation line segments between the nodal points (but not at the nodal points), as shown by the white arrows in Fig. 7, which enables these variants to have higher nucleus density along an individual dislocation line at lower $\theta_{m}$ (Fig. 4(a)).

\subsection{Effect of inclination angle between habit plane and GB dislocation line during growth}

During growth $\alpha$ precipitates encounter frequent coalescence and impingement events because of the high nucleation density along the dislocation lines (Fig. 2). The frequency of such coalescence or impingement events varies with the inclination angle between the fast growth directions (confined within the habit plane) of a variant and the dislocation lines. Figure 8 shows the inclination angle $\varphi_{\mathrm{hp}}$ between the habit plane of each variant and the GB dislocation lines for both tilt (Fig. 8(a)) and twist (Fig. 8(b)) GBs. Note that for the tilt GB, $\varphi_{\text {hp }}$ does not depend on $\theta_{m}$, since the dislocation line is parallel to the rotation axis $\left(/ / z[101]_{\beta}\right)$. While for the twist GBs, we calculate the inclination angle $\varphi_{\mathrm{hp}}$ between the habit planes of variants in $\beta_{1}$ and dislocation array $\xi_{1}$, as well as that between the habit planes of variants in $\beta_{2}$ and dislocation array $\xi_{2}$, since variants in $\beta_{1}$ and $\beta_{2}$ are preferred solely by dislocation array $\xi_{1}$ and $\xi_{2}$, respectively.

These calculation results show that variants having their habit planes parallel to the dislocation lines (i.e., $\varphi_{\mathrm{hp}}$ close to 0 ) grow preferentially along the dislocation lines, coalesce and form $\alpha$ laths. Depending on the inclination angles between the habit planes of these laths and the GBP, they could emanate from the GBP and grow into the interiors of the $\beta$ grains and form a typical colony of WS, or grow along the GBP and form a continuous GB $\alpha$ layer. For example, V7( $\left.\beta_{1}\right)$ and $\operatorname{V1}\left(\beta_{2}\right)$ in the case of tilt GBs have their habit planes parallel to the dislocation lines $\left(\varphi_{\mathrm{hp}}=0^{\circ}\right)$ and hence grow faster into the $\beta$ grains, free of interference from other variants, leading to the formation of WS (see Figs. 2 (b), (d), (f) and (h)). Besides, V9/V11 $\left(\beta_{1}\right)$ and V10/V12( $\left.\beta_{2}\right)$ in the 
case of the twist GBs have their habit planes deviate from $\xi_{1}$ and $\xi_{2}$, respectively, by a small angle $\left(\varphi_{\mathrm{hp}}=1^{\circ}\right)$ and thus behave similarly during their growth (see Fig. 3). On the other hand, V3 and V5 $\left(\varphi_{\mathrm{hp}}=29^{\circ}\right)$ in the case of twist GBs could grow along dislocations as well, but with a different manner, i.e., spreading over the GBP as Layer-I rather than growing into the interior of the $\beta$ grains. For the twist GB with $\theta_{m}=10^{\circ}$, the high density and uniform distribution of nuclei of V2 and V8 $\left(\varphi_{\mathrm{hp}}=33^{\circ}\right)$ formed at the dislocation intersections (nodal points) (Fig. 4(e)) result in extensive coalescence events along the two sets of dislocation lines, which enables V2 and V8 to expand at the GBP and form GB $\alpha$ Layer-II.

On the contrary, variants whose habit planes form large inclination angles with the dislocations do not have the same growth and coalescence advantage discussed above and their growth is confined by the neighboring variants that grow simultaneously in the same fashion. One example is V4 and V6 at tilt GBs shown in Fig. 2, whose habit planes have large inclination angles with the dislocation lines $\left(\varphi_{\mathrm{hp}}=57^{\circ}\right)$. Therefore, nucleation density, spatial distribution of nuclei, and inclination angle between habit plane of precipitate and GB dislocation line all have significant effects on the coalescence and impingement events during precipitate growth and, thus, on the VS process.

\subsection{Competition between GB $\alpha$ and WS as a function of misorientation angle}

The above analysis focuses on the competition among precipitates formed along the same dislocation line during their nucleation and growth. With the increase in misorientation angle $\theta_{m}$, however, one variant could also interact with other variants favored by neighboring dislocation segments due to the decrease in inter-dislocation spacing. For example, the morphology of $\alpha$ precipitates changes gradually from a mixture of equiaxed $\mathrm{GB} \alpha$ and individual WS to WS colony when $\theta_{m}$ increases from $3.5^{\circ}$ to $10^{\circ}$ (see Figs. 2(b), (d), (f), (h)). As discussed earlier, WS have the growth advantage over the other variants as they grow quickly into the $\beta$ grains and block the growth of the other variants. The decrease in inter-dislocation spacing suggests that there is not much inter-dislocation open space available for the GB $\alpha$ particles and WS (such as V9 in Figs. 2 (a) (c) and (e)) (nucleated preferably at $\omega=236^{\circ}$ or $213^{\circ}$ ) to grow. As a result, they tend to 
dissolve (due to their small sizes) during later stages, as indicated by the formation of the Widmanstätten colony structure in the case of large angle $\left(\theta_{m}=10^{\circ}\right)$ tilt GB. The variation of precipitate morphology at $\tau=10$ as a function of $\theta_{m}$ is schematically demonstrated in Fig. 9(a). With increasing $\theta_{m}$, the volume fraction of equiaxed GB $\alpha$ between dislocations decreases, while the volume fraction of the WS increases, which has been confirmed by our simulation data shown in Fig. 9(b). Note that an individual side-plate with two colors simply means that the two variants represented by the two colors have equal probability to form that side-plate and is not a composite side-plate with two variants.

The transition in precipitate morphology with increasing $\theta_{m}$ in the case of twist GBs is characterized by not only the variation of relative amount of $\alpha$ precipitates spreading along the GBP and those growing into $\beta$ grains as WS, but also the type of GB $\alpha$ layer, i.e., Layer-I and Layer-II. Layer-I is most preferred at a twist angle of $2^{\circ}$. However, with the decrease of inter-dislocation spacing (or increase of $\theta_{m}$ ), together with the decrease of distance between two neighboring line segments of dislocations $\xi_{1}$ and $\xi_{2}$, the growth of variants favored at the segments (i.e., those consisting of Layer-I) are significantly hindered as compared to those found in the case of $\theta_{m}=2^{\circ}$. Meanwhile, the density of dislocation nodal points (nucleation sites for V2 and V8) increases, which enhances the precipitation of V2 and V8, as shown in Fig. 4(e)-(h). The two types of GB $\alpha$ layer, i.e., Layer-I consisting of V3/V5 in $\beta_{1}$ and V4/V6 in $\beta_{2}$, and Layer-II consisting of V2/V8 in both grains, show opposite variation trends in volume fractions with increasing $\theta_{m}$, as shown in Fig. 10(a). The variation in the volume fraction of WS is also shown in Fig. 10(b). It suggests that for twist GBs when $\theta_{m} \leq 5^{\circ}$, the volume fraction of WS and hence $\mathrm{GB} \alpha$ layers remain relatively unchanged with approximately $1: 1$ ratio until $\theta_{m}$ approaches $5^{\circ}$ where the GB $\alpha$ layers start to dominate the precipitate morphology (Fig. 3). It should also be noted that due to the decrease in inter-dislocation spacing, the nucleation sites of WS near the twist GBs will increase accordingly, which leads to the formation of more WS of V12 (see Fig. 4(h)). However, these WS do not aggregate into colonies. This is because when the misorientation increases, variants preferred at the dislocation nodal points start to dominate and evolve into GB $\alpha$ layers via extensive coalescence events. The GB $\alpha$ layer gradually spreads over the GBP and 
isolates the WS into individual side-plates.

\subsection{Comparison between the simulation results and predictions from existing empirical rules}

\subsubsection{Effects of GBP orientation on VS}

As mentioned in the Introduction, some existing variant selection rules for the formation of GB $\alpha$ are based on the inclination angles between the habit planes of $\alpha$ variants and the GBP, such as Rule I: the angle between the low-energy interface of GB $\alpha$ and the GBP should be as small as possible and Rule II: the growth direction of preferred variants should be parallel or nearly parallel to the GBP. Obviously these rules ignore the discreteness of internal GB structures and rely solely on the geometry at the continuum level. Our current study shows that internal dislocation structures of GBs can have a significant effect on VS. To demonstrate this, the preferred variants of GB $\alpha$ during phase field simulations are compared with those predicted by Rule I and Rule II. Take $\beta_{1}$ as an example, the variants whose habit planes $\left(\{111113\}_{\beta}\right)$ or growth directions $\left(\langle 335\rangle_{\beta}\right)$ form minimum inclination angle with the GBP are listed in Table 2, corresponding to the prediction based on Rule I or Rule II, respectively. For the tilt GBs with different misorientation angle $\theta_{m}$, the empirical rules predict that the preferred $\mathrm{GB} \alpha$ are V1 by Rule I and V4/V6 by Rule II. In contrast, our simulation results show that the GB $\alpha$ selected by the GBs are V3/V4/V5/V6 for $\theta_{m}=3.5^{\circ} \sim 8.8^{\circ}$, among which V4 and V6 are consistent with those predicted by Rule II, but V3 and V5 are not predicted by either of the rules.

This discrepancy arises from the fact that the discrete internal dislocation structures at GBs distinguish further different nucleation sites for different variants and, thus, the preferred variants may not necessarily be consistent with the mean-field approach that treats GBs as uniform and homogeneous dividing surfaces. As seen clearly from Fig. 2, with the increase of $\theta_{m}$, the volume fraction of GB $\alpha$ consisting of V3/V4/V5/V6 decreases and almost vanishes at $\theta_{m}=10^{\circ}$. Such variation in the relative amounts of variants cannot be predicted by the simple empirical rules. For example, intuitively GB $\alpha$ should tend to grow along the GBP, in particular when the habit planes of the GB $\alpha$ are parallel to the GBP and we do observe the growth of GB $\alpha$ consisting of V4/V6 along the tilt GBP at $\theta_{m}=3.5^{\circ}$ (Fig. 2 (a)). However, when the discreteness of GB is considered, such growth will be interrupted by other variants whose habit planes may not be parallel to the 
GBP but contain the dislocation lines in the GBP, for example, by WS of V7 (Fig. 2(c) and (d)).

For the twist GBs, it should be noted that the inclination angles between the GBP and the habit planes of $\alpha$ variants will not change, because both the rotation axis and the normal of the GBP are parallel to the $z$ axis. We observe in the simulations that V3 and V5 are the preferred variants, which always obeys Rule I regardless of the misorientation angles. However, the presence of V2 and V8 when $\theta_{m}>5^{\circ}$ again violates both Rule I and Rule II. The above analysis suggests that the empirical rules may lose their validity because they ignore the intrinsic discreteness of GB structures and dynamic interactions among variants during nucleation and growth, which have been shown to play important roles in the current study by the phase field simulations.

\subsubsection{Relationship between GBa and WS}

Our simulation shows that for WS, those with the largest deviation of habit planes from the GBP are most likely to be selected. These variants include V7/V10/V12 $\left(\beta_{1}\right)$ and V1/V9/V11 $\left(\beta_{2}\right)$ for the tilt GBs, whose habit plane inclination angle $\lambda_{\mathrm{GB}}$ with the GBP reaches $\sim 50^{\circ}$, which varies slightly with $\theta_{m}$, and V9/V11 $\left(\beta_{1}\right)$ and V10/V12 $\left(\beta_{2}\right)$ for the twist GBs with $\lambda_{\mathrm{GB}}=86^{\circ}$. These WS arrange themselves either as isolated side-plates or as Widmanstätten colonies, which have been observed frequently in Ti-alloys. Different from what has been suggested in previous studies $[5,7,17]$, i.e., WS nucleate and grow from $\beta / \mathrm{GB} \alpha$ interfaces, the WS observed in the current study are formed directly at bare $\beta$ GBs. Even though we do observe some small WS that appear to be in contact with GB $\alpha$ from 2-D cross-sections (see Figs. 5(d) and (f)), they actually emanate directly from the GB in 3-D. As shown in the 2-D cross-sections (bottom row of Fig. 11(b), viewing direction $/ / z[101]_{\beta}$ ) of a 3-D simulation result (Figs. 11(a) and top row of 11(b)) in the case of a twist GB with $\theta_{m}=2^{\circ}$, although V10 seems to nucleate from the broad face of GB $\alpha$ V4, it actually nucleates directly at the GB at the same time when V4 is nucleated (i.e., at $\tau=2$ ), as can be seen from the corresponding 3-D images. The 2-D image of formation of V10 on the broad face of V4 turns out to be a typical 2-D sectioning artifact.

\subsection{Model assumptions and limitations}

Several assumptions made in the current study need to be noted. First of all, the intrinsic 
dislocation networks that characterize the GB structures are currently assumed to be composed of parallel arrays of Volterra dislocations [56]. This excludes possible dissociation of the dislocations into partials and dislocation nodal reactions [46]. In reality, whether or not certain dislocation nodal reactions can occur requires much more complicated calculations even for simple cases (e.g., symmetric tilt GBs) [55] and is indeed a theoretical challenge so far. To account for this effect on the stress field of the dislocation network, the microscopic phase field model of dislocation network at GBs [54] should be used. Secondly, only small angle special grain boundaries are considered in the current study because only the structures of low-angle and special GBs can be reasonably well modeled by dislocation networks. As an alternative, structural discreteness and the corresponding local stress fields of an arbitrary GB could be obtained from atomistic calculations [75] and then serve as inputs to the phase field model. This will allow one to explore effect of structures of arbitrary GB on $\alpha$ precipitation. For GBs with high angles, a terraced structure [76], on top of dislocation networks, may be present and result in additional discreteness. It is then expected that an irregular GB $\alpha$ layer with likely kinks/steps formed at the $\beta / \mathrm{GB} \alpha$ interfaces, owing to the much "rougher" GB structure and the resulting stress field. Those irregularities (usually concentrated with stresses) at $\beta / \mathrm{GB} \alpha$ interfaces may serve as nucleation sites for WS variants. But in our present study of the low-angle GBs, such a phenomenon or the so-called "auto-catalytic effects" between $\mathrm{GB} \alpha$ and WS is not observed. Thirdly, it also needs to be pointed out that experimentally observed GB $\alpha$ and WS are usually formed under continuous cooling rather than isothermal aging as in our current study. The dynamically varying driving force may further facilitate and promote WS to emanate from existing irregular $\beta / \mathrm{GB} \alpha$ interfaces. Therefore, in all cases, the actual discrete structure of GBs should play a key role in determining the subsequent precipitate morphology. Finally, only mechanical effect from the GB (e.g., the stress field of GB dislocation networks) is considered and possible chemical effects (e.g., segregation and possible segregation transition at dislocations and GBs $[77,78]$ ) are ignored.

\section{Conclusions}

The effects of intrinsic grain boundary (GB) structure on morphology and variant selection (VS) during $\alpha$ precipitation at low-angle $\beta$ GBs in Ti-6Al-4V are investigated systematically using phase field simulations. Symmetrical tilt and pure twist GBs are considered and their structures 
are modeled as dislocation networks. It is found that the formation of two distinctive types of $\alpha$ precipitates at or near a GB frequently observed in experiments, i.e., GB allotriomorphs (GB $\alpha$ ) and Widmanstätten side-plates (WS), together with the underlying VS, correlate highly with (i) elastic interaction between a nucleating $\alpha$ precipitate and the GB dislocation network, (ii) growth direction relative to the dislocation line directions, (iii) density of preferred nucleation sites for the same variant and coalescence during growth and (iv) spatial confinement from simultaneously nucleated neighboring $\alpha$ variants of dissimilar types. Detailed findings include:

- For the tilt $\mathrm{GBs}, \mathrm{GB} \alpha$ are mainly comprised of individual equiaxed particles between neighboring dislocations, which are preferred mainly at small misorientation angles $\left(\theta_{m} \leq 3.5^{\circ}\right)$ and disappear gradually as the misorientation angle increases. For the twist GBs, GB $\alpha$ consists mainly of $\alpha$ layer of two groups, V3/V5 in $\beta_{1}$ or V4/V6 in $\beta_{2}\left(29^{\circ}\right.$ inclination between their habit planes and the corresponding dislocation lines) and V2/V8 (33 inclination between their habit planes and the dislocation lines). The volume fraction of the former group decreases and that of the latter group increases as the misorientation angle increases.

- The WS are found to form directly from GB dislocations. The volume fraction of WS formed at the tilt GBs shows a positive correlation with the misorientation angle from $\theta_{m}=3.5^{\circ}$ to $\theta_{m}=10^{\circ}$, while it reaches maximum at $\theta_{m}=3^{\circ}$ in one grain and at $\theta_{m}=5^{\circ}$ in the other at the twist GBs.

- The degree of VS of a given variant is determined by 1) the strength of its elastic interaction with the GB dislocations, 2) number of other variants sharing the same preferred nucleation sites, and 3) preponderant growth direction relative to the GB dislocation line directions. Factors 1) and 2) dominate VS mainly during nucleation while factor 3) affects VS mainly during growth. At the same time, the misorientation angle will affect the precipitation process in both stages, i.e., the density of nuclei at the nucleation stage and the competition among variants near neighboring dislocations at the growth stage.

Even though only symmetrical tilt and pure twist GBs are considered in the current study, the major findings about the effect of GB structure on precipitate morphology and VS should be general for special GBs that have well-defined defect structures. 


\section{Acknowledgements}

This work is supported by the 973 Programs under Grants No. 2012CB619600 and No. 2014CB644003, National Nature Science Foundation of China under Grant No. 51504151 (D.Q., D.Z. and W.L) and NSF DMREF program under Grant No. DMR-1435483 (R.P.S and Y.W).

\section{References}

[1] G.C. Obasi, S. Birosca, J.Q. da Fonseca, M. Preuss. Effect of $\beta$ grain growth on variant selection and texture memory effect during $\alpha \rightarrow \beta \rightarrow \alpha$ phase transformation in Ti-6 Al-4 V, Acta Materialia 60 (2012) 1048.

[2] G.C. Obasi, S. Birosca, D.G.L. Prakash, J.Q. da Fonseca, M. Preuss. The influence of rolling temperature on texture evolution and variant selection during $\alpha \rightarrow \beta \rightarrow \alpha$ phase transformation in Ti6Al-4V, Acta Materialia 60 (2012) 6013.

[3] D. Banerjee, J.C. Williams. Perspectives on Titanium Science and Technology, Acta Materialia 61 (2013) 844.

[4] S.L. Semiatin, K.T. Kinsel, A.L. Pilchak, G.A. Sargent. Effect of Process Variables on Transformation-Texture Development in Ti-6Al-4V Sheet Following Beta Heat Treatment, Metallurgical and Materials Transactions A 44 (2013) 3852.

[5] B. Appolaire, L. Héricher, E. Aeby-Gautier. Modelling of phase transformation kinetics in Ti alloys - Isothermal treatments, Acta Materialia 53 (2005) 3001.

[6] E. Aeby-Gautier, F. Bruneseaux, J.D.C. Teixeira, B. Appolaire, G. Geandier, S. Denis. Microstructural formation in Ti alloys: In-situ characterization of phase transformation kinetics, JOM 59 (2007) 54.

[7] J. Da Costa Teixeira, B. Appolaire, E. Aeby-Gautier, S. Denis, L. Héricher. Modeling of the phase transformations in near- $\beta$ titanium alloys during the cooling after forging, Computational Materials Science 42 (2008) 266.

[8] S.M.C. van Bohemen, A. Kamp, R.H. Petrov, L.A.I. Kestens, J. Sietsma. Nucleation and variant selection of secondary $\alpha$ plates in a $\beta$ Ti alloy, Acta Materialia 56 (2008) 5907.

[9] M. Salib, J. Teixeira, L. Germain, E. Lamielle, N. Gey, E. Aeby-Gautier. Influence of transformation temperature on microtexture formation associated with $\alpha$ precipitation at $\beta$ grain boundaries in a $\beta$ metastable titanium alloy, Acta Materialia 61 (2013) 3758.

[10] D. Bhattacharyya, G. Viswanathan, H.L. Fraser. Crystallographic and morphological relationships between $\beta$ phase and the Widmanstätten and allotriomorphic $\alpha$ phase at special $\beta$ grain boundaries in an $\alpha / \beta$ titanium alloy, Acta Materialia 55 (2007) 6765.

[11] R. Shi, V. Dixit, H.L. Fraser, Y. Wang. Variant selection of grain boundary $\alpha$ by special prior $\beta$ grain boundaries in titanium alloys, Acta Materialia 75 (2014) 156.

[12] R. Banerjee, D. Bhattacharyya, P.C. Collins, G.B. Viswanathan, H.L. Fraser. Precipitation of grain boundary $\alpha$ in a laser deposited compositionally graded Ti-8Al-xV alloy - an orientation microscopy study, Acta Materialia 52 (2004) 377.

[13] G. Lütjering, J.J.C. Williams. Titanium, Springer, 2007.

[14] T. Ahmed, H.J. Rack. Phase transformations during cooling in $\alpha+\beta$ titanium alloys, Materials 
Science and Engineering: A 243 (1998) 206.

[15] D. Bhattacharyya, G. Viswanathan, R. Denkenberger, D. Furrer, H.L. Fraser. The role of crystallographic and geometrical relationships between $<\mathrm{i}>\alpha</ \mathrm{i}>$ and $<\mathrm{i}>\beta</ \mathrm{i}>$ phases in an $<\mathrm{i}>$ $\alpha</ \mathrm{i}>/<\mathrm{i}>\beta</ \mathrm{i}>$ titanium alloy, Acta materialia 51 (2003) 4679.

[16] E. Lee, R. Banerjee, S. Kar, D. Bhattacharyya, H.L. Fraser. Selection of $\alpha$ variants during microstructural evolution in $\alpha / \beta$ titanium alloys, Philosophical Magazine 87 (2007) 3615.

[17] Z. Sun, S. Guo, H. Yang. Nucleation and growth mechanism of $\alpha$-lamellae of Ti alloy TA15 cooling from an $\alpha+\beta$ phase field, Acta Materialia 61 (2013) 2057.

[18] V. Dixit. Grain-Boundary Parameters Controlled Allotriomorphic Phase Transformations in Beta-Processed Titanium Alloys. The Ohio State University, 2013.

[19] K. Ameyama, G.C. Weatherly, K.T. Aust. A study of grain boundary nucleated widmanstätten precipitates in a two-phase stainless steel, Acta metallurgica et materialia 40 (1992) 1835.

[20] I. Lischewski, G. Gottstein. Nucleation and variant selection during the $\alpha-\gamma-\alpha$ phase transformation in microalloyed steel, Acta Materialia 59 (2011) 1530.

[21] N. Stanford, P.S. Bate. Crystallographic variant selection in Ti-6Al-4V, Acta Materialia 52 (2004) 5215 .

[22] G. Lütjering. Influence of processing on microstructure and mechanical properties of $(\alpha+\beta)$ titanium alloys, Materials Science and Engineering: A 243 (1998) 32.

[23] D. Banerjee, J. Williams. Perspectives on Titanium Science and Technology, Acta Materialia 61 (2013) 844.

[24] W. Burgers. On the process of transition of the cubic-body-centered modification into the hexagonal-close-packed modification of zirconium, Physica 1 (1934) 561.

[25] R. Shi, N. Ma, Y. Wang. Predicting equilibrium shape of precipitates as function of coherency state, Acta Materialia 60 (2012) 4172.

[26] N. Gey, M. Humbert, M. Philippe, Y. Combres. Modeling the transformation texture of Ti-64 sheets after rolling in the $\beta$-field, Materials Science and Engineering: A 230 (1997) 68.

[27] T. Furuhara, T. Maki. Variant selection in heterogeneous nucleation on defects in diffusional phase transformation and precipitation, Materials Science and Engineering: A 312 (2001) 145.

[28] N. Gey, E. Gautier, M. Humbert, A. Cerqueira, J. Bechade, P. Archambault. Study of the $\alpha / \beta$ phase transformation of Zy-4 in presence of applied stresses at heating: analysis of the inherited microstructures and textures, Journal of nuclear materials 302 (2002) 175.

[29] N. Gey, M. Humbert. Characterization of the variant selection occurring during the $\alpha \rightarrow \beta \rightarrow \alpha$ phase transformations of a cold rolled titanium sheet, Acta materialia 50 (2002) 277.

[30] L. Germain, N. Gey, M. Humbert, P. Bocher, M. Jahazi. Analysis of sharp microtexture heterogeneities in a bimodal IMI 834 billet, Acta materialia 53 (2005) 3535.

[31] M. Humbert, L. Germain, N. Gey, P. Bocher, M. Jahazi. Study of the variant selection in sharp textured regions of bimodal IMI 834 billet, Materials Science and Engineering: A 430 (2006) 157.

[32] S. Wang, M. Aindow, M. Starink. Effect of self-accommodation on $<\mathrm{i}>\alpha</ \mathrm{i}>\mid<\mathrm{i}>\alpha</ \mathrm{i}>$ boundary populations in pure titanium, Acta materialia 51 (2003) 2485.

[33] T. Furuhara, S. Takagi, H. Watanabe, T. Maki. Crystallography of grain boundary a precipitates in a $\beta$ titanium alloy, Metallurgical and Materials Transactions A 27 (1996) 1635.

[34] D. Bhattacharyya, G.B. Viswanathan, R. Denkenberger, D. Furrer, H.L. Fraser. The role of crystallographic and geometrical relationships between $\alpha$ and $\beta$ phases in an $\alpha / \beta$ titanium alloy, Acta Materialia 51 (2003) 4679. 
[35] P.J. Clemm, J.C. Fisher. The influence of grain boundaries on the nucleation of secondary phases, Acta Metallurgica 3 (1955) 70.

[36] J.W. Cahn. The kinetics of grain boundary nucleated reactions, Acta Metallurgica 4 (1956) 449.

[37] K.C. Russell. Grain boundary nucleation kinetics, Acta Metallurgica 17 (1969) 1123.

[38] J.K. Lee, H.I. Aaronson. Influence of faceting upon the equilibrium shape of nuclei at grain boundaries-I. Two-dimensions, Acta metallurgica 23 (1975) 799.

[39] J.K. Lee, H.I. Aaronson. Influence of faceting upon the equilibrium shape of nuclei at grain boundaries-II. Three-dimensions, Acta Metallurgica 23 (1975) 809.

[40] R. Shi, V. Dixit, G.B. Viswanathan, H.L. Fraser, Y. Wang. Experimental assessment of variant selection rules for grain boundary $\alpha$ in titanium alloys, Acta Materialia 102 (2016) 197.

[41] L.J. Brillson. The structure and properties of metal-semiconductor interfaces, Surface Science Reports 2 (1982) 123.

[42] M.J. Demkowicz, J. Wang, R.G. Hoagland. Interfaces between dissimilar crystalline solids, Dislocations in solids 14 (2008) 141.

[43] R.G. Hoagland, R.J. Kurtz. The relation between grain-boundary structure and sliding resistance, Philosophical Magazine A 82 (2002) 1073.

[44] K. Kolluri, M.J. Demkowicz. Dislocation mechanism of interface point defect migration, Physical Review B 82 (2010) 193404.

[45] R.W. Siegel, S.M. Chang, R.W. Balluffi. Vacancy loss at grain boundaries in quenched polycrystalline gold, Acta Metallurgica 28 (1980) 249.

[46] A.P. Sutton, R.W. Balluffi. Interfaces in crystalline materials, (1995).

[47] W. Luo, C. Shen, Y. Wang. Nucleation of ordered particles at dislocations and formation of split patterns, Acta materialia 55 (2007) 2579.

[48] H. Liu, Y. Gao, Y.M. Zhu, Y. Wang, J.F. Nie. A simulation study of $\beta 1$ precipitation on dislocations in an Mg-rare earth alloy, Acta Materialia 77 (2014) 133.

[49] D. Qiu, R. Shi, D. Zhang, W. Lu, Y. Wang. Variant selection by dislocations during a precipitation in $\alpha / \beta$ titanium alloys, Acta Materialia 88 (2015) 218.

[50] B.A. Bilby. Report on the conference on defects in crystalline solids, The Physical Society, London 123 (1955).

[51] F.C. Frank. Report of the symposium on the plastic deformation of crystalline solids, Carnegie Institute of Technology, Pittsburgh (1950) 150.

[52] W.-Z. Zhang. Calculation of Interfacial Dislocation Structures: Revisit to the O-lattice Theory, Metallurgical and Materials Transactions A 44 (2013) 4513.

[53] W.Z. Zhang. Formulas for periodic dislocations in general interfaces, Applied Physics Letters 86 (2005) 121919.

[54] C. Shen, J. Li, Y. Wang. Predicting structure and energy of dislocations and grain boundaries, Acta Materialia 74 (2014) 125.

[55] J.P. Hirth, J. Lothe. Theory of dislocations, John Willey and Sons, New York, 1982.

[56] A.J. Vattré, M.J. Demkowicz. Determining the Burgers vectors and elastic strain energies of interface dislocation arrays using anisotropic elasticity theory, Acta Materialia 61 (2013) 5172.

[57] R. Shi, N. Zhou, S.R. Niezgoda, Y. Wang. Microstructure and transformation texture evolution during $\alpha$ precipitation in polycrystalline $\alpha / \beta$ titanium alloys - A simulation study, Acta Materialia 94 (2015) 224.

[58] R. Shi, Y. Wang. Variant selection during $\alpha$ precipitation in Ti-6Al-4V under the influence of 
local stress - A simulation study, Acta Materialia 61 (2013) 6006.

[59] J.W. Cahn, J.E. Hilliard. Free Energy of a Nonuniform System. I. Interfacial Free Energy, The Journal of Chemical Physics 28 (1958) 258.

[60] A.G. Khachaturian. Theory of structural transformations in solids, 1983.

[61] Y.U. Wang, Y.M. Jin, A.G. Khachaturyan. Phase field microelasticity theory and modeling of elastically and structurally inhomogeneous solid, Journal of Applied Physics 92 (2002) 1351.

[62] I. Steinbach, F. Pezzolla, B. Nestler, M. Seeßelberg, R. Prieler, G.J. Schmitz, J.L.L. Rezende. A phase field concept for multiphase systems, Physica D: Nonlinear Phenomena 94 (1996) 135.

[63] I. Steinbach, F. Pezzolla. A generalized field method for multiphase transformations using interface fields, Physica D: Nonlinear Phenomena 134 (1999) 385.

[64] D. Hull, D.J. Bacon. Introduction to dislocations, Pergamon Press Oxford, 1984.

[65] A.J. Vattre, N. Abdolrahim, K. Kolluri, M.J. Demkowicz. Computational design of patterned interfaces using reduced order models, Sci Rep 4 (2014) 6231.

[66] C. Shen, J. Simmons, Y. Wang. Effect of elastic interaction on nucleation: II. Implementation of strain energy of nucleus formation in the phase field method, Acta materialia 55 (2007) 1457.

[67] W.W. Mullins, R.F. Sekerka. Morphological stability of a particle growing by diffusion or heat flow, Journal of applied physics 34 (1963) 323.

[68] W.W. Mullins, R.F. Sekerka. Stability of a planar interface during solidification of a dilute binary alloy, Journal of applied physics 35 (1964) 444.

[69] I. Loginova, J. Ågren, G. Amberg. On the formation of Widmanstätten ferrite in binary Fe-Cphase-field approach, Acta Materialia 52 (2004) 4055.

[70] Y.-Z. Wang, N. Ma, Q. Chen, F. Zhang, S. Chen, Y. Chang. Predicting phase equilibrium, phase transformation, and microstructure evolution in titanium alloys, JOM 57 (2005) 32.

[71] J. Tiley, T. Searles, E. Lee, S. Kar, R. Banerjee, J.C. Russ, H.L. Fraser. Quantification of microstructural features in $\alpha / \beta$ titanium alloys, Materials Science and Engineering: A 372 (2004) 191.

[72] D. Banerjee, A.L. Pilchak, J.C. Williams. Processing, Structure, Texture and Microtexture in Titanium Alloys. vol. 710: Trans Tech Publ, 2012. p.66.

[73] J.P. Hirth. A brief history of dislocation theory, Metallurgical Transactions A 16 (1985) 2085.

[74] R. Peierls. The size of a dislocation, Proceedings of the Physical Society 52 (1940) 34.

[75] X.-Y. Sun, V. Taupin, C. Fressengeas, P. Cordier. Continuous description of the atomic structure of grain boundaries using dislocation and generalized-disclination density fields, International Journal of Plasticity 77 (2016) 75.

[76] D.G. Brandon. The structure of high-angle grain boundaries, Acta metallurgica 14 (1966) 1479.

[77] N. Ma, S.A. Dregia, Y. Wang. Solute segregation transition and drag force on grain boundaries, Acta Materialia 51 (2003) 3687.

[78] N. Ma, C. Shen, S.A. Dregia, Y. Wang. Segregation and wetting transition at dislocations, Metallurgical and Materials Transactions A 37 (2006) 1773.

\section{Figure captions:}

Fig. 1. Schematic illustrations of grain boundary (GB) structure characterized by dislocation network for (a) symmetrical tilt and (b) pure twist GB. The rotation axis in both cases are [101 $]_{\beta}$ (// Z-axis), with the Burgers vector $\mathbf{b}$ and sense vector $\xi$ of the resulting dislocations being listed as well. In (b), $\phi$ denotes the inclination angle between two sets of dislocations. 
Fig. 2. Precipitation sequence of $\alpha$ phase near symmetrical tilt GBs with different misorientation angles: (a-b) $\theta_{m}=3.5^{\circ}$, (c-d) $\theta_{m}=7.0^{\circ}$, (e-f) $\theta_{m}=8.8^{\circ}$, and (g-h) $\theta_{m}=10^{\circ}$. Different $\alpha$ variants are colored according to the color bar and the white lines indicate the edge of GBP represented as the light gray plane. A reduced simulation time $\tau=1$ corresponds to a real time of $t=10^{-4} \mathrm{~s}$.

Fig. 3. Morphology and variant selection of $\alpha$ precipitates near twist GBs with different misorientation angles: (a) $\theta_{m}=2^{\circ}$, (b) $\theta_{m}=3^{\circ}$, (c) $\theta_{m}=5^{\circ}$ and (d) $\theta_{m}=10^{\circ}$ at simulation time $\tau=10$. Each row has three different viewing directions to highlight $\alpha$ precipitates in $\beta_{1}, \beta_{2}$ and near GB.

Fig. 4. Time evolution of $\alpha$ precipitates near a twist GB of (a-d) $\theta_{m}=2^{\circ}$ on the side of $\beta_{1}$ and (e-h) $\theta_{m}=10^{\circ}$ on the side of $\beta_{2}$, showing the formation of WS in company with two different types of GB $\alpha$ layers, i.e. Layer-I and Layer-II. The Layer-II in (e-h) can extend along GBP in either 1-D mode (within the white ellipses) or 2-D mode (within the yellow circles).

Fig. 5. Morphologies of $\alpha$ variants near twist GB of different $\theta_{m}$ in two dimensions obtained through cross-sectioning three-dimensional microstructure in (a-c) by slicing planes (light blue) that are perpendicular to the GBP, as shown in (d-f) with edge-on view along GBP, respectively. The white lines indicate the intersection between the GBP and slice planes.

Fig. 6. Minimum interaction energies between variants in $\beta_{1}$ (green bars) / $\beta_{2}$ (red bars) and the stress field of a symmetrical tilt GB with $\theta_{m}=3.5^{\circ}$.

Fig. 7. (a) Minimum interaction energies between variants in $\beta_{1}$ (green bars) / $\beta_{2}$ (red bars) and the stress field of a twist GB with $\theta_{m}=2^{\circ}$. The distribution of interaction energy and preferred nucleation sites (indicated by the open rectangles and arrows) of some selected variants near GBP are shown on the side of (b) $\beta_{1}$ grain and (c) $\beta_{2}$ grain.

Fig. 8. Inclination angles $\varphi_{\mathrm{hp}}$ between the habit plane of each variant and the dislocation line in the case of the (a) tilt and (b) twist GB. Note that for the tilt GB, inclination angles $\varphi_{\mathrm{hp}}$ do not change for variants in different $\beta$ grains, since the dislocation line is parallel to the rotation axis (//Z-axis) and for twist GB, only $\varphi_{\mathrm{hp}}$ between variants in $\beta_{1}$ and the dislocation $\xi_{1}$, and that between variants in $\beta_{2}$ and $\xi_{2}$ are calculated.

Fig. 9. (a) Schematic illustrations of the dependence of precipitate morphology (i.e. competition between GB $\alpha$ and Widmanstätten side-plates) near symmetrical tilt GBs on misorientation angle $\left(\theta_{m}\right)$ based on the simulation results at $\tau=10$, (b) the corresponding volume fraction of side-plates.

Fig. 10. Misorientation angle $\left(\theta_{m}\right)$ dependence of the volume fraction of (a) GB $\alpha$ layer and (b) WS in both grains near twist GBs. Note that in the former both Layer-I (consisting of V3/V5 in $\beta_{1}$ and V4/V6 in $\beta_{2}$ ) and Layer-II (consisting of V2/V8 in both $\beta$ grains) are calculated separately in each grain.

Fig. 11. A comparison between 2-D observations and 3-D microstructure of GB $\alpha$ : (a) the full 3-D microstructure near twist GB at $\tau=2$ with GBP indicated by the gray plane and cross section by the blue plane, (b) the enlarged 2-D and 3-D microstructures from the circled region in (a) evolving from $\tau=2$ to $\tau=10$. The white line in (a) 
and (b) indicates the projection of cross section into the GBP.

\section{Table captions}

Table 1 . The relative position of preferred nucleation site (characterized by $\omega$ ) around an edge dislocation of a symmetrical tilt GB for all the $\alpha$ variants in the two adjacent $\beta$ grains

Table 2. Comparison between variant selection of GB $\alpha$ in $\beta_{1}$ predicted by Rule I and Rule II with that observed in the phase field simulations. 

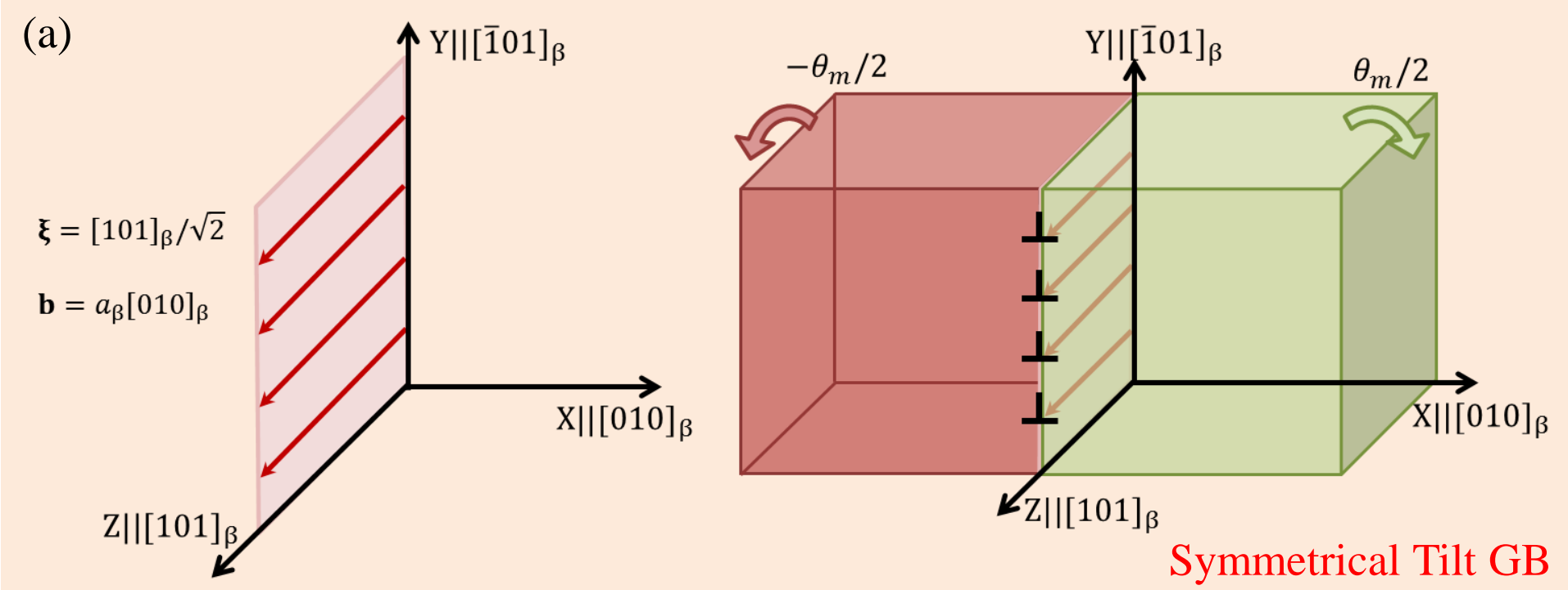

(b)
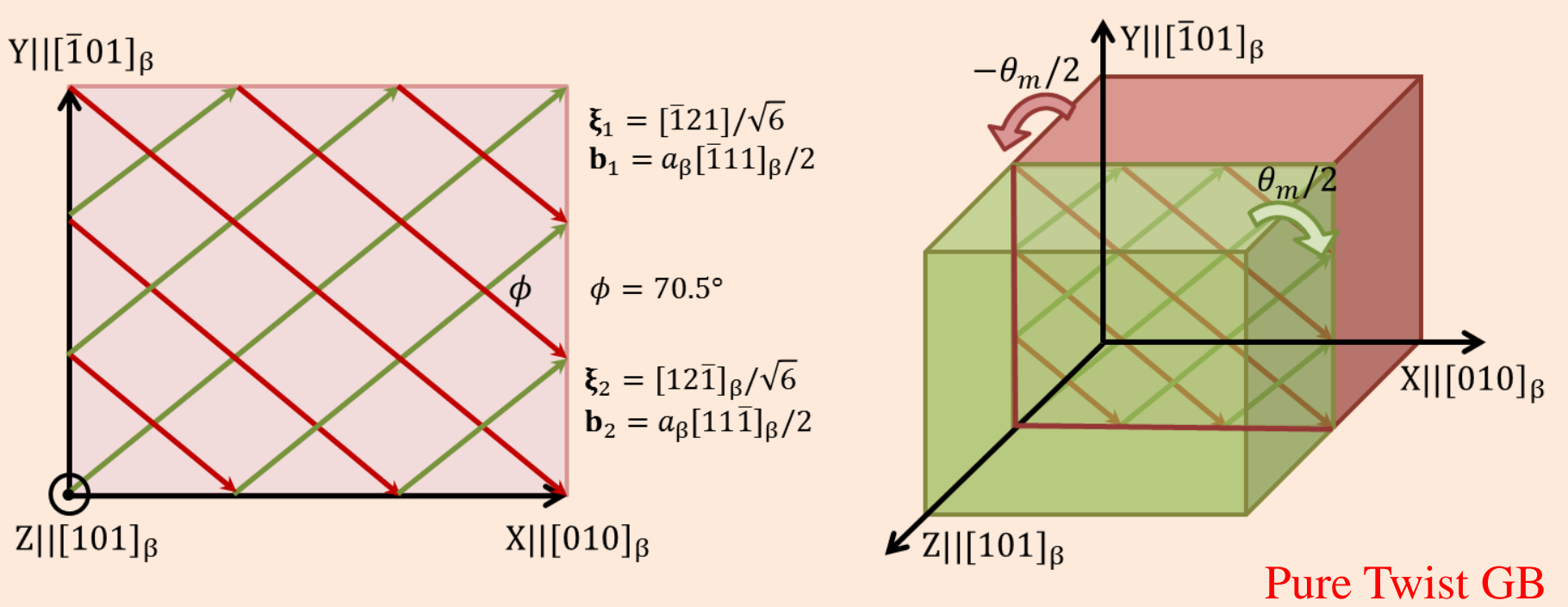

Fig. 1 

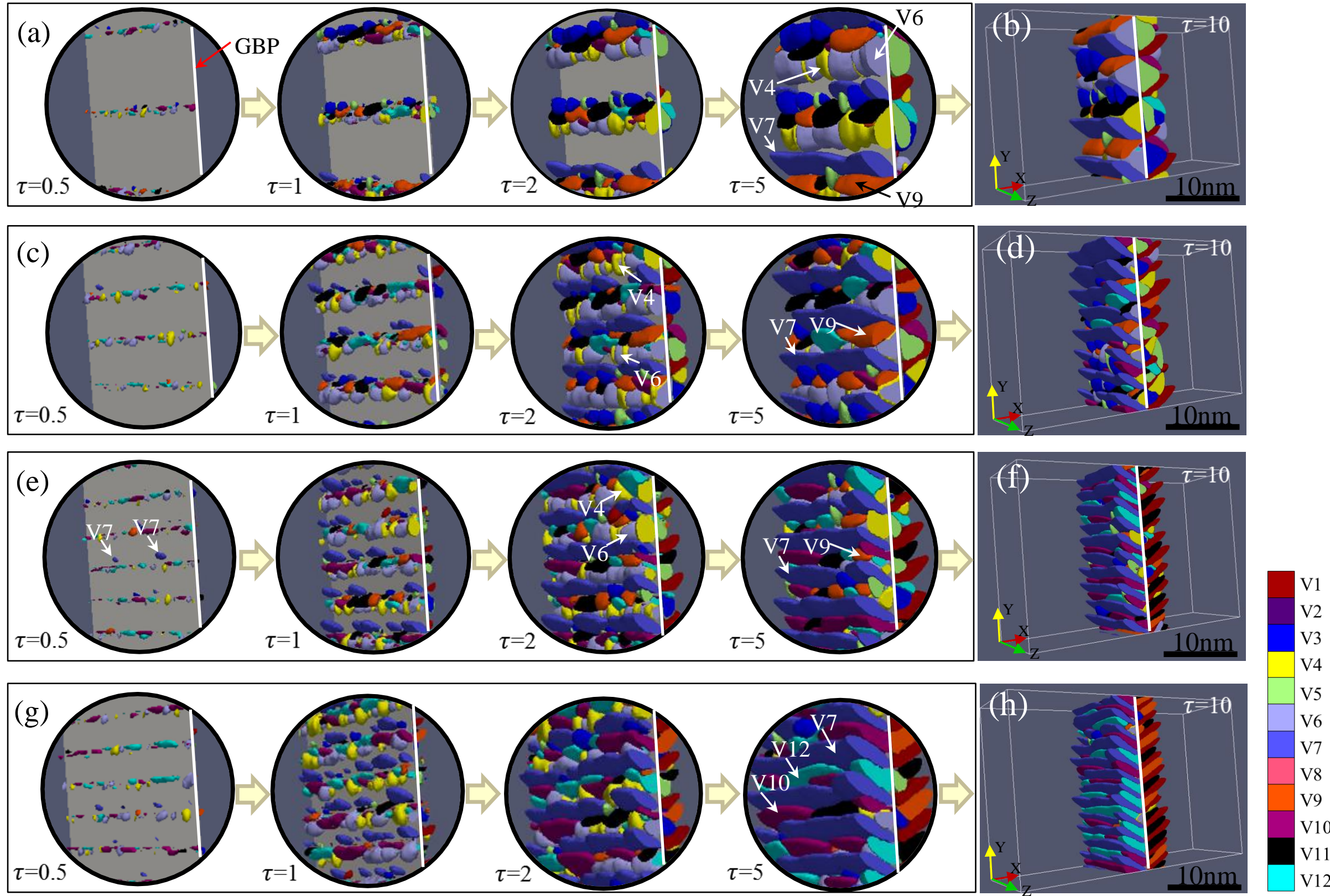

Fig. 2 


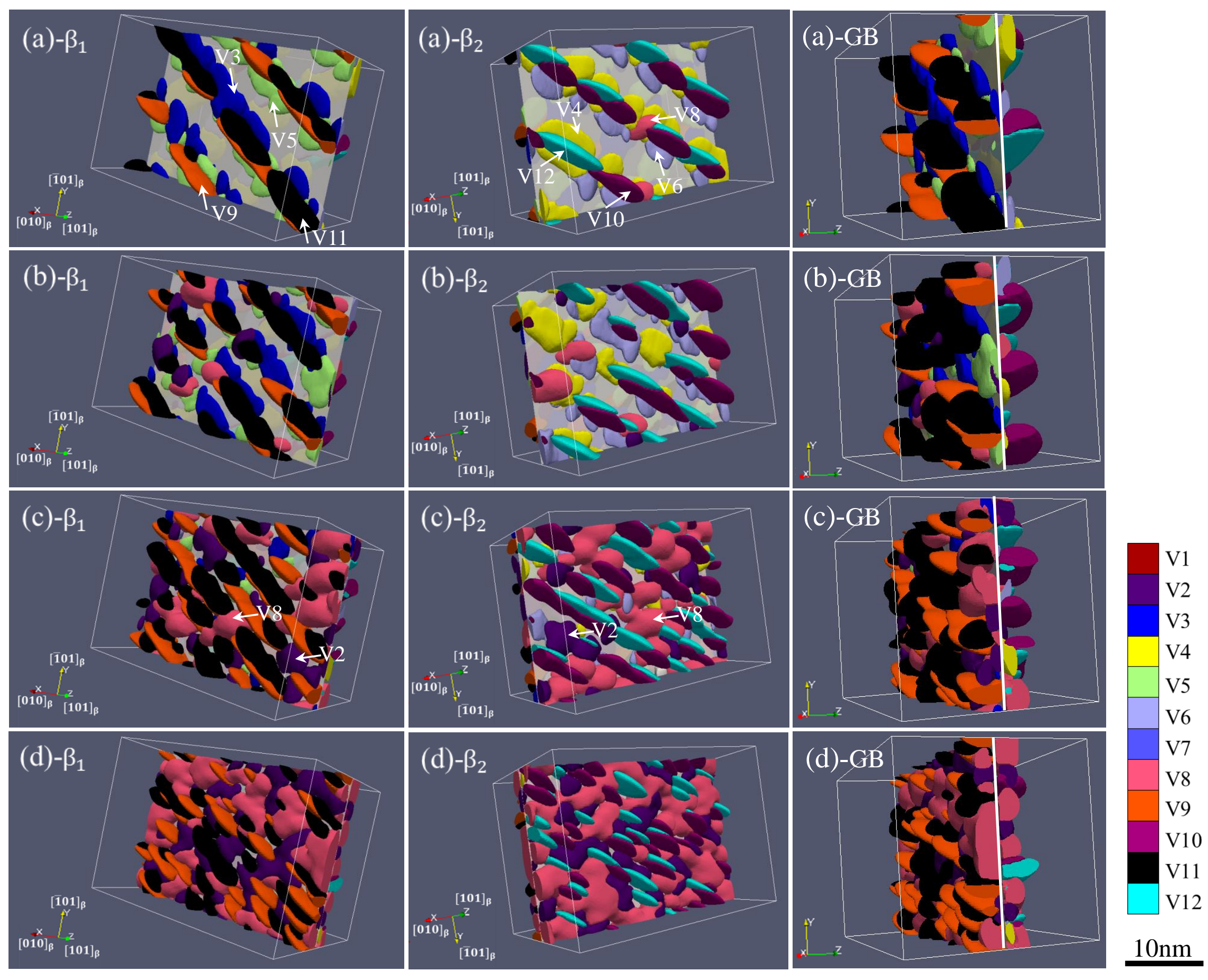




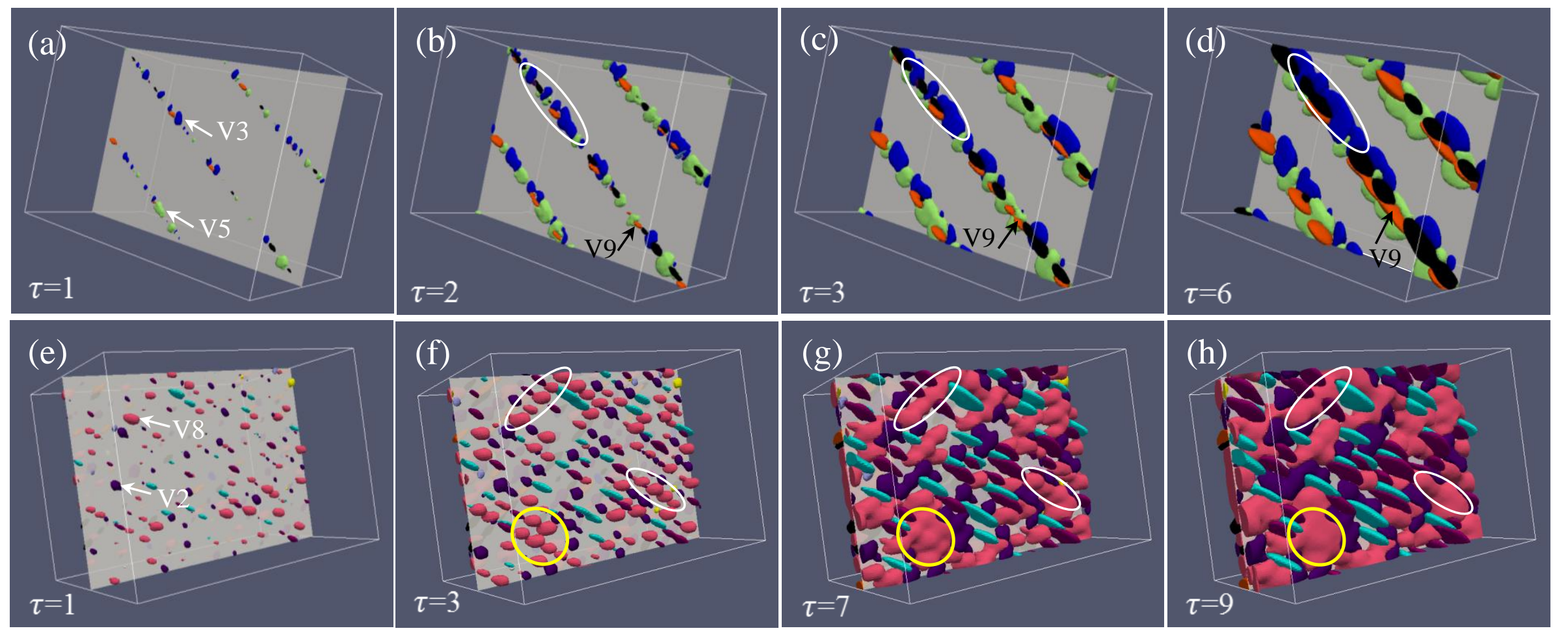

Fig. 4 


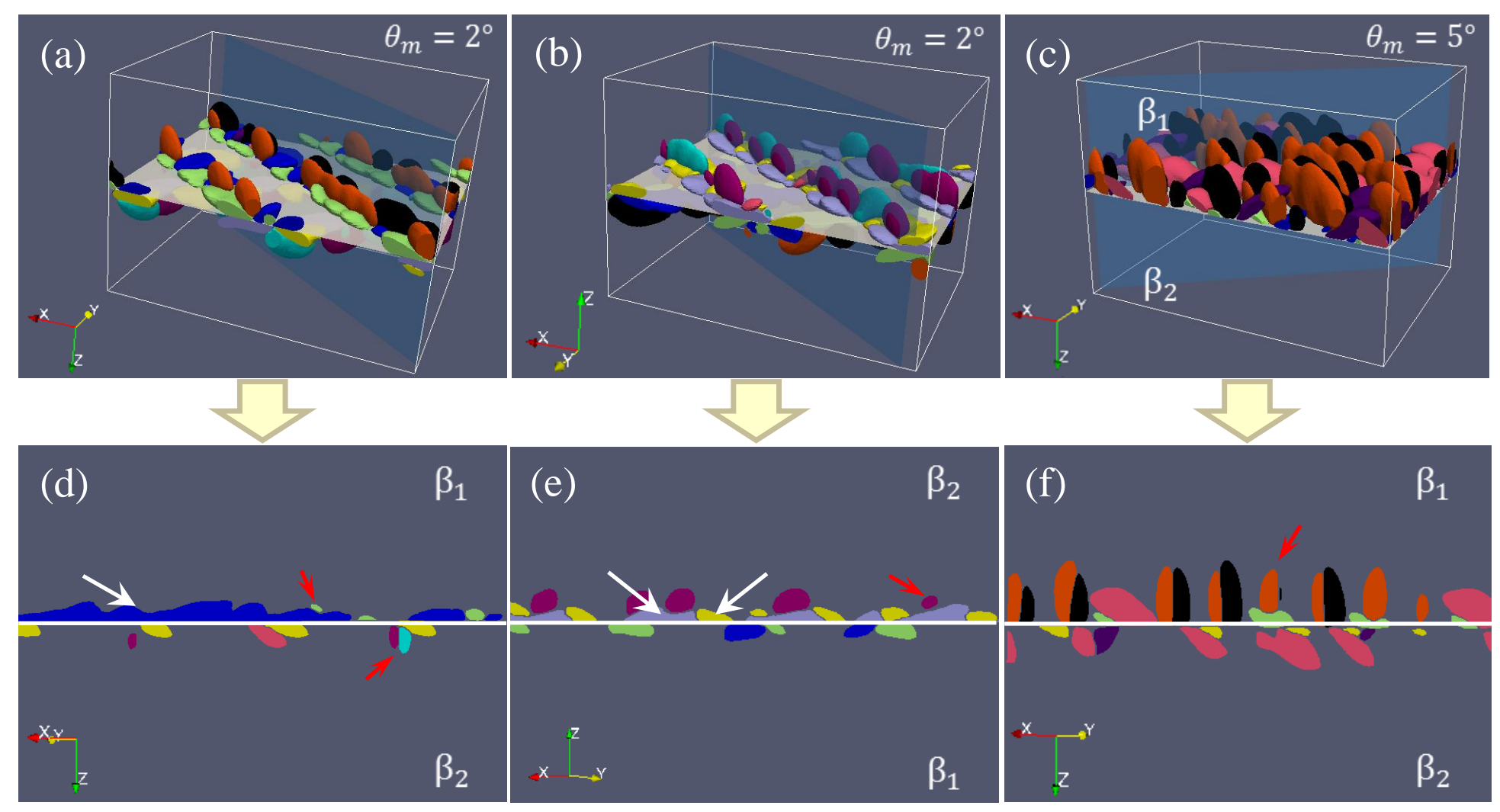

Fig. 5 


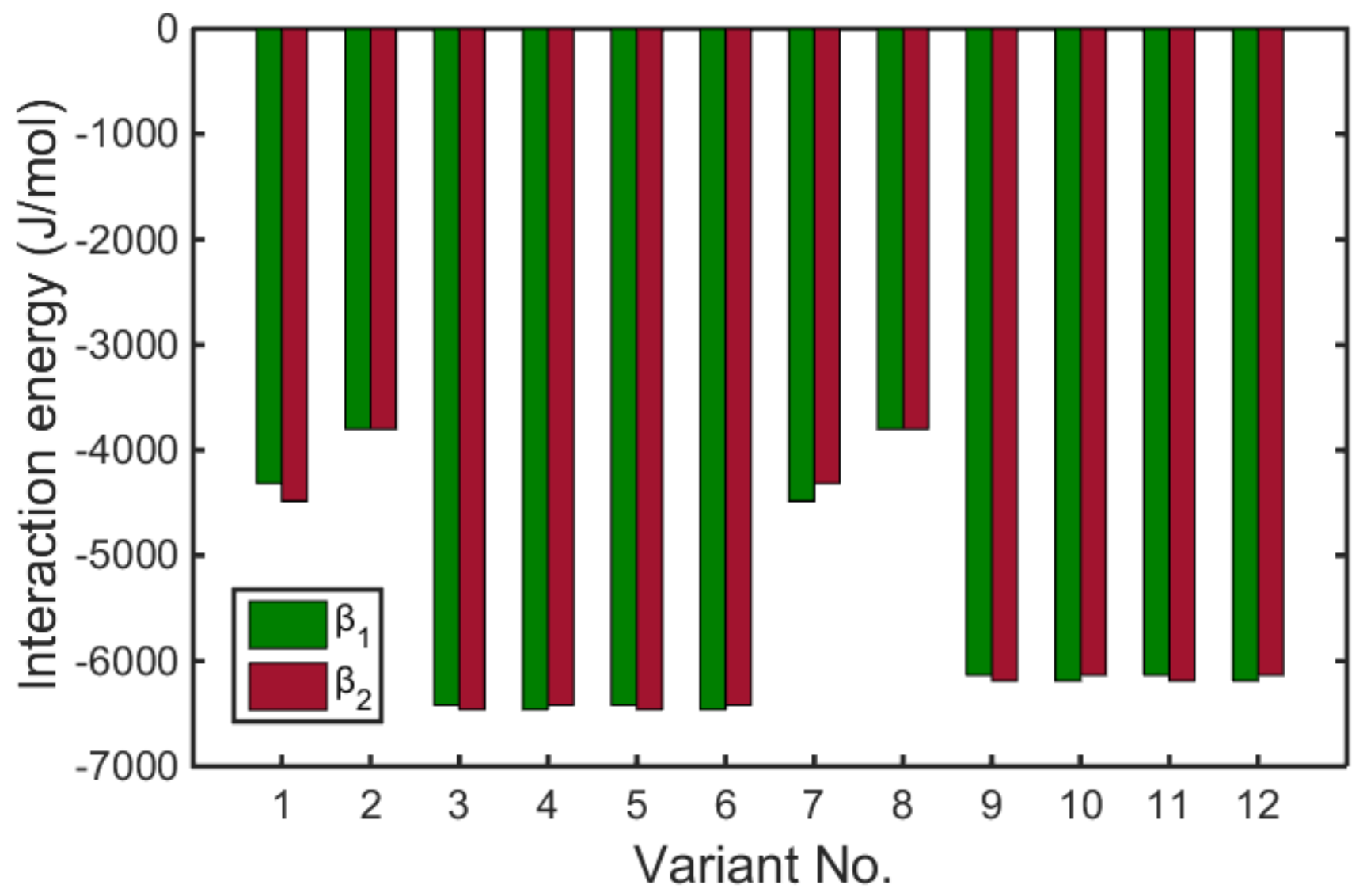

Fig. 6 

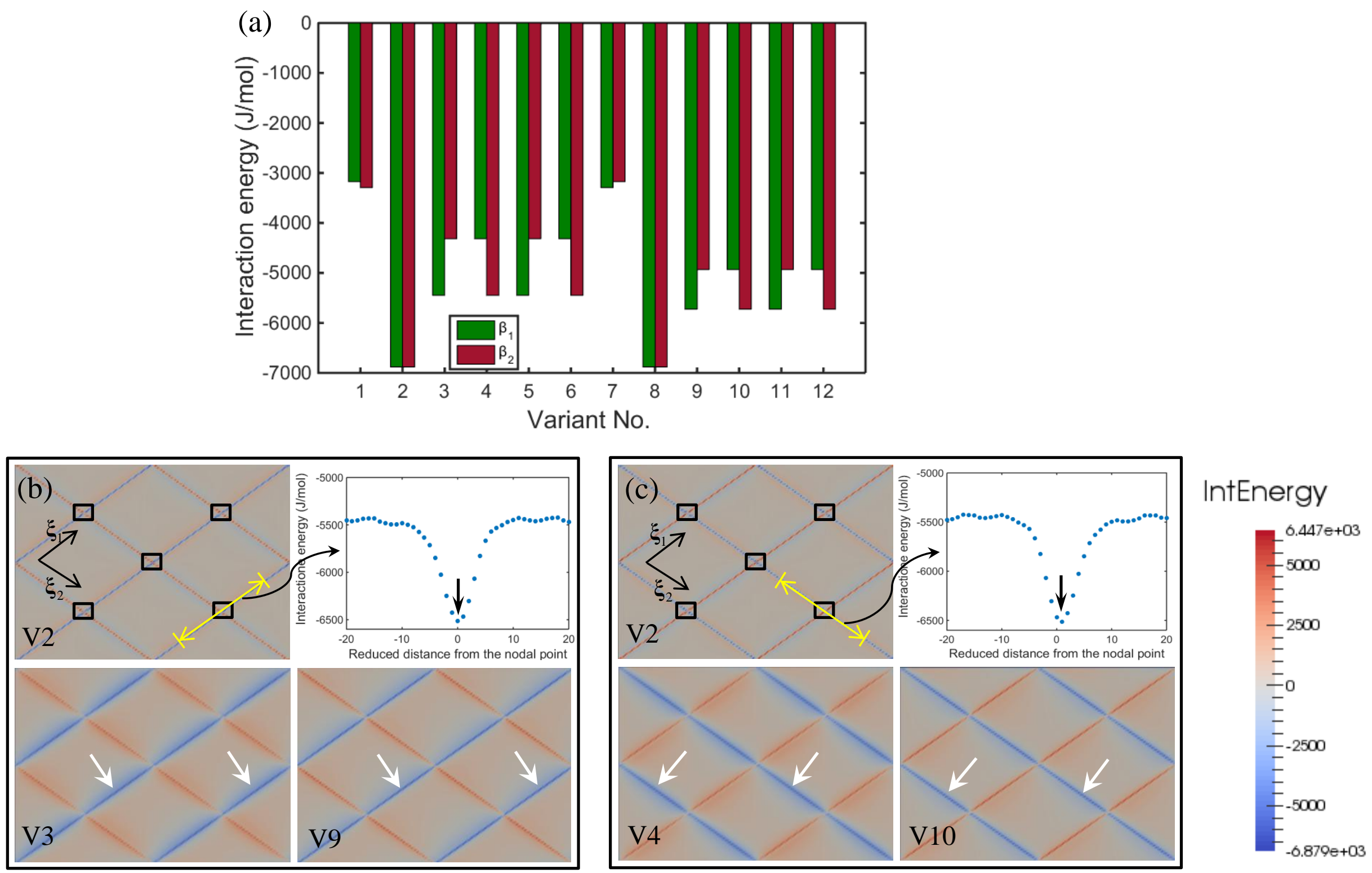

Fig. 7 

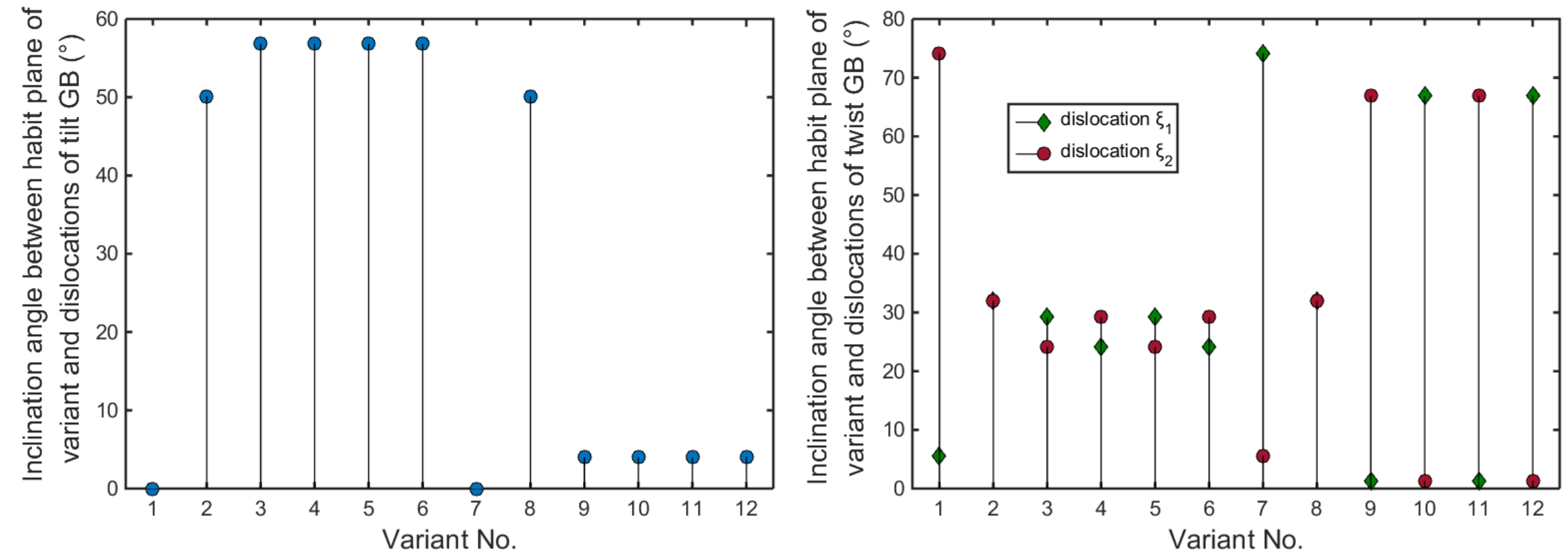

Fig. 8 

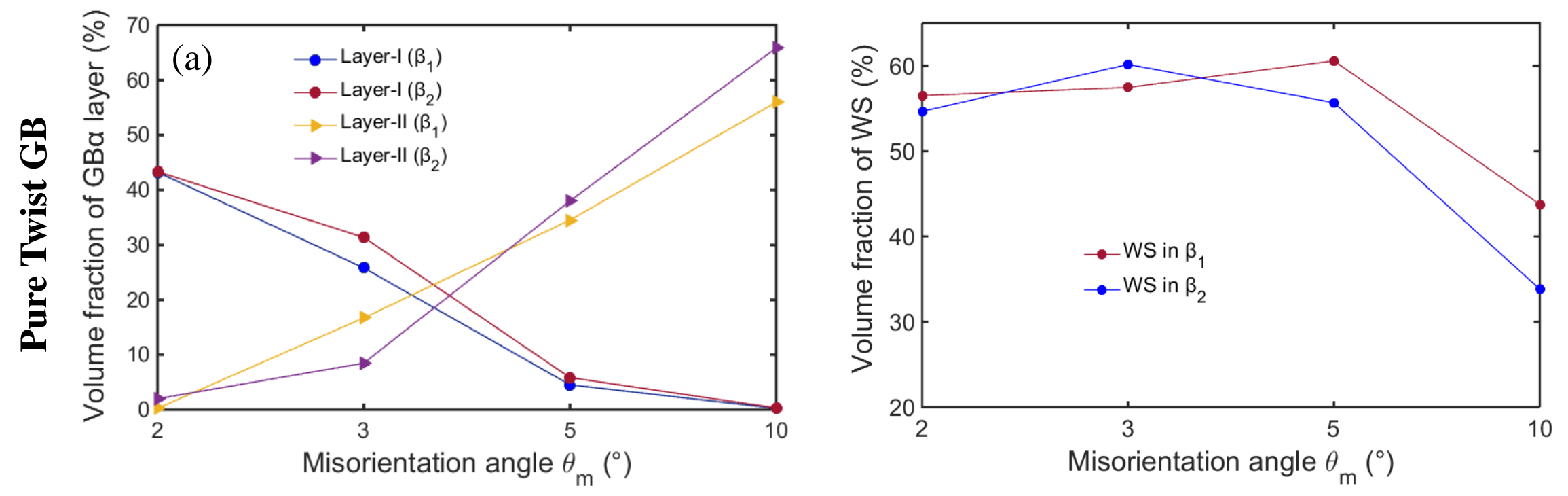

Fig. 10 
Table 1 . The relative position of preferred nucleation site (characterized by $\omega$ ) around an edge dislocation of a symmetrical tilt GB for all the $\alpha$ variants in the two adjacent $\beta$ grains

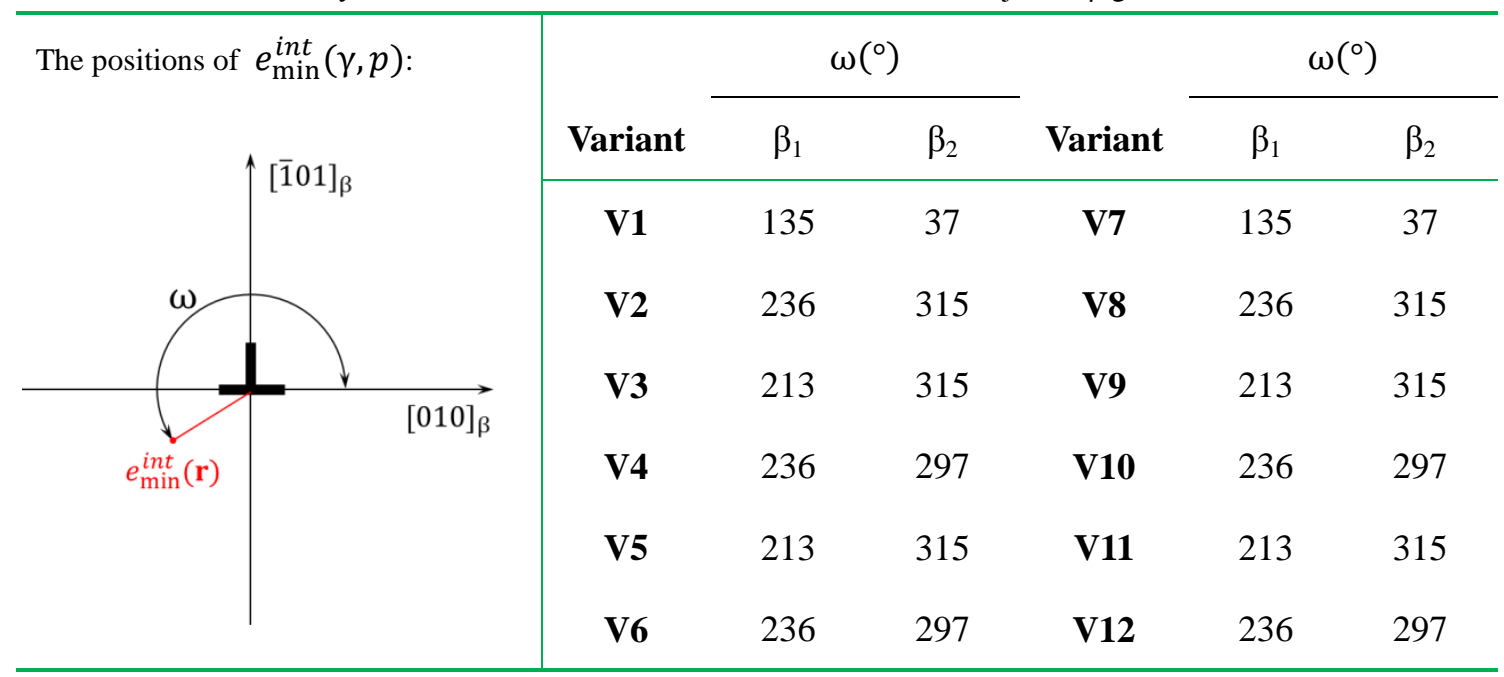

Table 2. Comparison between variant selection of GB $\alpha$ in $\beta_{1}$ predicted by Rule I and Rule II with that observed in the phase field simulations

\begin{tabular}{c|cccc}
\hline GB type & $\theta_{m}$ & Rule I & Rule II & Simulation Results \\
\hline \multirow{2}{*}{ Tilt } & $3.5^{\circ}$ & $\mathrm{V} 1$ & $\mathrm{~V} 4, \mathrm{~V} 6$ & $\mathrm{~V} 3, \mathrm{~V} 4, \mathrm{~V} 5, \mathrm{~V} 6$ \\
& $7.0^{\circ}$ & $\mathrm{V} 1$ & $\mathrm{~V} 4, \mathrm{~V} 6$ & $\mathrm{~V} 3, \mathrm{~V} 4, \mathrm{~V} 5, \mathrm{~V} 6$ \\
& $8.8^{\circ}$ & $\mathrm{V} 1$ & $\mathrm{~V} 4, \mathrm{~V} 6$ & $\mathrm{~V} 3, \mathrm{~V} 4, \mathrm{~V} 5, \mathrm{~V} 6$ \\
& $10.0^{\circ}$ & $\mathrm{V} 1$ & $\mathrm{~V} 4, \mathrm{~V} 6$ & Mainly WS colonies \\
\hline Twist & $-*$ & $\mathrm{~V} 3, \mathrm{~V} 4, \mathrm{~V} 5, \mathrm{~V} 6$ & $\mathrm{~V} 1, \mathrm{~V} 7$ & $\mathrm{~V} 3, \mathrm{~V} 5, \mathrm{~V} 2, \mathrm{~V} 8$ \\
\hline
\end{tabular}

* The relative orientation between variants and twist GB is invariable with the change of $\theta_{m}$. 


\section{Graphical Abstract}

\section{Symmetrical tilt} grain boundary

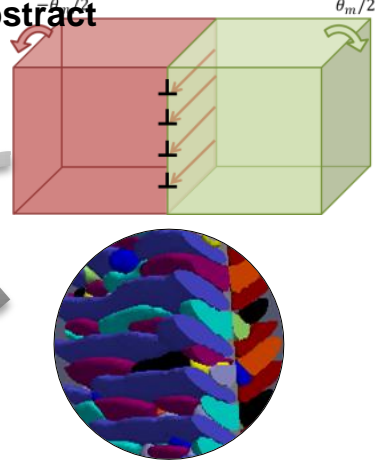

Pure twist grain boundary

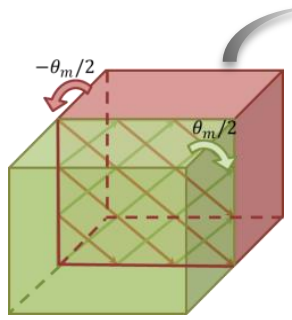

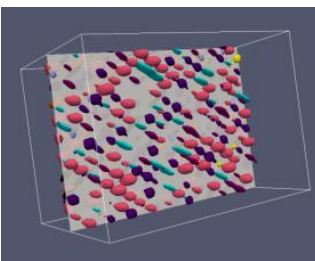

